\title{
Conformal mapping for cavity inverse problem: an explicit reconstruction formula
}

\author{
Alexandre Munnier* Karim Ramdani ${ }^{\dagger}$
}

June 29, 2021

\begin{abstract}
In this paper, we address a classical case of the Calderón (or conductivity) inverse problem in dimension two. We aim to recover the location and the shape of a single cavity $\omega$ (with boundary $\gamma$ ) contained in a domain $\Omega$ (with boundary $\Gamma$ ) from the knowledge of the Dirichlet-to-Neumann (DtN) map $\Lambda_{\gamma}:\left.f \longmapsto \partial_{n} u^{f}\right|_{\Gamma}$, where $u^{f}$ is harmonic in $\Omega \backslash \bar{\omega},\left.u^{f}\right|_{\Gamma}=f$ and $\left.u^{f}\right|_{\gamma}=c^{f}, c^{f}$ being the constant such that $\int_{\gamma} \partial_{n} u^{f} \mathrm{~d} s=0$. We obtain an explicit formula for the complex coefficients $a_{m}$ arising in the expression of the Riemann map $z \longmapsto a_{1} z+a_{0}+\sum_{m \leqslant-1} a_{m} z^{m}$ that conformally maps the exterior of the unit disk onto the exterior of $\omega$. This formula is derived by using two ingredients: a new factorization result of the DtN map and the so-called generalized Pólia-Szegö tensors (GPST) of the cavity. As a byproduct of our analysis, we also prove the analytic dependence of the coefficients $a_{m}$ with respect to the DtN. Numerical results are provided to illustrate the efficiency and simplicity of the method.
\end{abstract}

\section{Introduction}

Let $\Omega$ be a simply connected open bounded set in $\mathbb{R}^{2}$ with Lipschitz boundary $\Gamma$. Let $\sigma$ be a positive function in $L^{\infty}(\Omega)$ and consider the elliptic boundary value problem:

$$
\begin{aligned}
-\nabla \cdot(\sigma \nabla u) & =0 & & \text { in } \Omega \\
u & =f & & \text { on } \Gamma .
\end{aligned}
$$

Calderón's inverse conductivity problem [15] can be stated as follows: Knowing the Dirichlet-toNeumann (DtN) map $\Lambda_{\gamma}: f \longmapsto \partial_{n} u^{f}$, is it possible to recover the conductivity $\sigma$ ?

In this work, we focus on the particular case of piecewise conductivity with infinitely high contrast (see for instance Friedman and Vogelius [19] who considered this problem in the case of small inclusions). More precisely, we suppose that $\Omega$ contains a cavity $\omega$, where $\omega$ is an open connected set with Lipschitz boundary $\gamma$ and such that $\bar{\omega} \subset \Omega$ (see Figure 1). We denote by $n$ the unit normal to $\Gamma \cup \gamma$ directed towards the exterior of $\Omega \backslash \bar{\omega}$.

\footnotetext{
*Université de Lorraine and CNRS, Institut Élie Cartan de Lorraine, UMR 7502, Vandœuvre-lès-Nancy, F-54506, France. alexandre.munnier@univ-lorraine.fr

${ }^{\dagger}$ Inria, Villers-lès-Nancy, F-54600, France. karim.ramdani@inria.fr
} 


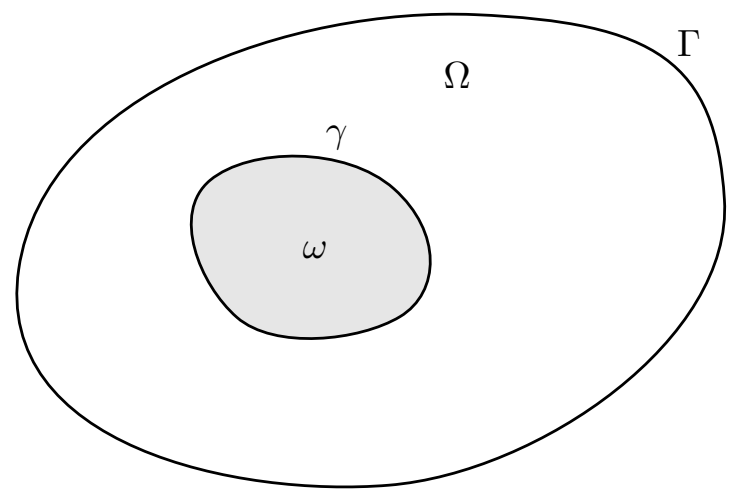

Figure 1: The geometry.

For every $f$ in $H^{\frac{1}{2}}(\Gamma)$, we denote by $\left(u^{f}, c^{f}\right) \in H^{1}(\Omega \backslash \bar{\omega}) \times \mathbb{R}$ the solution to the Dirichlet problem:

$$
\begin{array}{rlrl}
-\Delta u^{f}=0 & & \text { in } \Omega \backslash \bar{\omega} \\
u^{f}=f & & \text { on } \Gamma \\
u^{f} & =c^{f} & & \text { on } \gamma,
\end{array}
$$

where $c^{f}$ is the unique constant such that:

$$
\int_{\gamma} \partial_{n} u^{f} \mathrm{~d} \sigma=0
$$

Problem (1.2) is well-posed and its solution is the limit of the solution of 1.1 for piecewise constant conductivity, when the contrast between the cavity and the background tends to infinity (see Proposition A.1 of the Appendix for a precise statement of this classical result and for the proof, which is given for the sake of completeness).

Loosely speaking (the exact functional framework will be made precise later on), the inverse problem considered throughout this paper is the following: knowing the Dirichlet-to-Neumann (DtN) map $\Lambda_{\gamma}: f \longmapsto \partial_{n} u^{f}$, how to reconstruct the cavity $\omega$ ?

Remark 1.1 In dimension 2, it is classical to see $u^{f}$ as the harmonic conjugate function of $v^{f}$, i.e. the solution to:

$$
\begin{aligned}
-\Delta v^{f} & =0 & & \text { in } \Omega \backslash \bar{\omega} \\
\partial_{n} v^{f} & =\partial_{\tau} f & & \text { on } \Gamma \\
\partial_{n} v^{f} & =0 & & \text { on } \gamma,
\end{aligned}
$$

where $\tau:=n^{\perp}$ is the unit tangent vector to $\Gamma$. The function $u^{f}$ is usually referred to as the stream function associated to the potential function $v^{f}$. On $\Gamma, \partial_{n} u^{f}=-\partial_{\tau} v^{f}$ and therefore, the knowledge of $\Lambda_{\gamma}$ (i.e. the DtN for $u^{f}$ ) is equivalent to the knowledge of the Neumann-to-Dirichlet map for $v^{f}$.

Classically for inverse problems, the questions of uniqueness, stability and reconstruction have been studied in the literature for cavities identification. Regarding uniqueness, it is well-known that 
one pair $\left(f, \partial_{n} u^{f}\right)$ of Cauchy data uniquely determines the geometry of the cavity for a Dirichlet boundary condition (see Kress [33]) or a Neumann boundary condition (see Alessandrini and Rondi [2]). For Robin type condition, Bacchelli [9] proved that two excitations $f_{1}$ and $f_{2}$ uniquely determine the cavity provided they are linearly independent and one of them is positive. Concerning stability, as shown by Mandache [36], logarithmic stability is best possible (see also Alessandrini and Rondi [2] and references therein). Among the reconstruction methods available in the literature for shape identification, one can distinguish two classes of approaches: iterative and non iterative methods (see for instance the survey paper by Potthast [39] for an overview of reconstruction methods). In the first class of methods, one computes a sequence of approximating shapes, generally by solving at each step the direct problem and using minimal data (typically only one or several pairs of Cauchy data, and not the full DtN map). Among these approaches, we can mention those based on optimization [10, 16], on the reciprocity gap principle [35, 31, 14], on the quasi-reversibility [11, 12] or on conformal mapping [1, 33, 20, 21, 22, 34, 23].

The second class of methods covers non iterative methods which are generally based on the construction (from the measurements) of an indicator function of the inclusion(s). These sampling/probe methods do not need to solve the forward problem, but require the knowledge of the full DtN map. Among these reconstruction techniques, let us mention -with no claim as to completeness- the enclosure and probe method of Ikehata [27, 29, 28, 30, 18, Kirsch's Factorization method [13, 24, 32] and Generalized Polya-Szegö Tensors in the case of small inclusions [6, 7, 8, 5].

Our purpose in this paper is to propose a new non iterative reconstruction method that combines some of the ingredients used in earlier works, namely: a new factorization result (Theorem 3.1), Generalized Polya-Szegö Tensors and conformal mapping. The main feature of our reconstruction method is that we end up with an explicit reconstruction formula (Theorem 3.4) for the complex coefficients $a_{k}$ arising in the expression of the Riemann map $z \longmapsto a_{1} z+a_{0}+\sum_{m \leqslant-1} a_{m} z^{m}$ that conformally maps the exterior of the unit disk onto the exterior of $\omega$. Let us emphasize that these reconstruction formulae also yield the analytic dependence of the coefficients with respect to the DtN.

The proposed reconstruction algorithm can -in principle- be adapted to other boundary conditions. However, such as most direct reconstruction methods, it requires the knowledge of the full DtN map and so far, it is limited to the two-dimensional case due to the use of conformal mapping.

The paper is organized as follows: we present in Section 2 a boundary integral formulation of the problem. Section 3 is devoted to the derivation of the reconstruction formula, using a new factorization result and GPST. Some issues about stability are also discusses therein. Finally, some numerical results are given in Section 4 .

\section{Boundary integral formulation}

\subsection{Background on single layer potential}

In this section, we collect some well known facts of potential theory, and more especially on single layer potential, that are crucial for our method. For more details and for the proofs, we refer the interested reader to the monographs of McLean [37, Steinbach [40] or Hsiao and Wendland [26].

Throughout the article, we shall denote by

$$
G(x)=-\frac{1}{2 \pi} \log |x|
$$

the fundamental solution of the operator $-\Delta$ in $\mathbb{R}^{2}$. 
Let $C_{i}$ be a bounded, Lipschitz domain (see [37, Definition 3.28]) and denote by $\mathscr{C}$ its boundary. Let $n$ be the unit normal to $\mathscr{C}$ directed towards the exterior of $\mathrm{C}_{i}$.

The exterior of $C_{i}$ is denoted $C_{e}:=\mathbb{R}^{2} \backslash \bar{C}_{i}$. Given a function $u$ in $H_{\ell o c}^{1}\left(\mathbb{R}^{2}\right)$, we denote by $u_{i}$ and $u_{e}$ its restrictions respectively to $\mathrm{C}_{i}$ and $\mathrm{C}_{e}$ and by $[u]_{\mathscr{C}}=\left.u_{i}\right|_{\mathscr{C}}-\left.u_{e}\right|_{\mathscr{C}} \in H^{\frac{1}{2}}(\mathscr{C})$ its jump across $\mathscr{C}$. We also define similarly the jump of the normal derivative: $\left[\partial_{n} u\right]_{\mathscr{C}}=\left.\left(\partial_{n} u_{i}\right)\right|_{\mathscr{C}}-\left.\left(\partial_{n} u_{e}\right)\right|_{\mathscr{C}} \in H^{-\frac{1}{2}}(\mathscr{C})$.

Definition 2.1 For every $\hat{q} \in H^{-\frac{1}{2}}(\mathscr{C})$, we denote by $\mathscr{S}_{\mathscr{C}} \hat{q}$ the single layer potential associated to the density $\hat{q}$.

The single layer potential $\mathscr{S}_{\mathscr{C}} \hat{q}$ defines a harmonic function in $\mathbb{R}^{2} \backslash \mathscr{C}$. The operator $\mathscr{S}_{\mathscr{C}}$ is an integral operator with weakly singular kernel, so that for $\hat{q} \in L^{\infty}(\mathscr{C})$ for instance and $x \in \mathbb{R}^{2} \backslash \mathscr{C}$, it reads:

$$
\mathscr{S}_{\mathscr{C}} \hat{q}(x)=\int_{\mathscr{C}} G(x-y) \hat{q}(y) \mathrm{d} \sigma_{y}
$$

Moreover, the single layer potential defines a bounded linear operator from $H^{-\frac{1}{2}}(\mathscr{C})$ into $H_{\text {loc }}^{1}\left(\mathbb{R}^{2}\right)$, and $\mathscr{S}_{\mathscr{C}} \hat{q}$ admits the following asymptotic behavior at infinity (see for instance [37, p. 261])

$$
\mathscr{S}_{\mathscr{C}} \hat{q}(x)=-\frac{1}{2 \pi}\langle\hat{q}, 1\rangle_{-\frac{1}{2}, \frac{1}{2}, \mathscr{C}} \log |x|+O\left(|x|^{-1}\right)
$$

where $\langle\cdot, \cdot\rangle_{-\frac{1}{2}, \frac{1}{2}, \mathscr{C}}$ stands for the duality brackets between $H^{-\frac{1}{2}}(\mathscr{C})$ and $H^{\frac{1}{2}}(\mathscr{C})$. This shows in particular that $\mathscr{S}_{\mathscr{C}} \hat{q} \in H^{1}\left(\mathbb{R}^{2}\right)$ if and only if $\hat{q}$ belongs to the function space

$$
\widehat{H}(\mathscr{C}):=\left\{\hat{q} \in H^{-\frac{1}{2}}(\mathscr{C}):\langle\hat{q}, 1\rangle_{-\frac{1}{2}, \frac{1}{2}, \mathscr{C}}=0\right\} .
$$

We also recall that the single layer potential satisfies the following classical jump conditions

$$
\left[\mathscr{S}_{\mathscr{C}} \hat{q}\right]_{\mathscr{C}}=0, \quad\left[\partial_{n}\left(\mathscr{S}_{\mathscr{C}} \hat{q}\right)\right]_{\mathscr{C}}=\hat{q}
$$

Let us focus now on the trace of the single layer potential.

Definition 2.2 For every $\hat{q} \in H^{-\frac{1}{2}}(\mathscr{C})$, we denote by $\mathrm{S}_{\mathscr{C}} \hat{q}$ the trace of the single layer operator $\mathscr{S}_{\mathscr{C}} \hat{q}$ on $\mathscr{C}$.

The operator $\mathrm{S}_{\mathscr{C}}$ is an integral operator with weakly singular kernel as well. For $\hat{q} \in L^{\infty}(\mathscr{C})$ and for every $x \in \mathscr{C}$, it reads:

$$
\mathrm{S}_{\mathscr{C}} \hat{q}(x)=\int_{\mathscr{C}} G(x-y) \hat{q}(y) \mathrm{d} \sigma_{y} .
$$

The trace $\mathrm{S}_{\mathscr{C}}$ of the single layer operator defines a bounded linear operator from $H^{-\frac{1}{2}}(\mathscr{C})$ into $H^{\frac{1}{2}}(\mathscr{C})$. Furthermore, using Green's formula and the asymptotics 2.1, we can easily prove the identity

$$
\left\langle\hat{q}, \mathrm{~S}_{\Gamma} \hat{q}\right\rangle_{-\frac{1}{2}, \frac{1}{2}, \mathscr{C}}=\int_{\mathbb{R}^{2}}\left|\nabla\left(\mathscr{S}_{\mathscr{C}} \hat{q}\right)\right|^{2} \mathrm{~d} x<+\infty, \quad \forall \hat{q} \in \widehat{H}(\mathscr{C}) .
$$

Following [37, Theorem 8.15], we also introduce the following particular density and constant which will play a crucial role in our analysis. 
Definition 2.3 The equilibrium density for $\mathscr{C}$ is the unique density $\hat{\mathrm{e}}_{\mathscr{C}} \in H^{-\frac{1}{2}}(\mathscr{C})$ such that $\mathrm{S}_{\mathscr{C}} \hat{\mathrm{e}}_{\mathscr{C}}$ is constant on $\mathscr{C}$ and

$$
\left\langle\hat{\mathrm{e}}_{\mathscr{C}}, 1\right\rangle_{-\frac{1}{2}, \frac{1}{2}, \mathscr{C}}=1 \text {. }
$$

The logarithmic capacity $\operatorname{Cap}(\mathscr{C})$ of $\mathscr{C}$ is defined as being the positive constant:

$$
\operatorname{Cap}(\mathscr{C})=\exp \left(-2 \pi \mathrm{S}_{\mathscr{C}} \hat{\mathrm{e}}_{\mathscr{C}}\right)
$$

Setting

$$
H(\mathscr{C}):=\left\{q \in H^{\frac{1}{2}}(\mathscr{C}):\left\langle\hat{\mathrm{e}}_{\mathscr{C}}, q\right\rangle_{-\frac{1}{2}, \frac{1}{2}, \mathscr{C}}=0\right\}
$$

we know, following McLean [37], that the linear operator:

$$
\mathrm{S}_{\mathscr{C}}: \hat{q} \in \widehat{H}(\mathscr{C}) \longmapsto q \in H(\mathscr{C})
$$

defines an isomorphism that extends into an isomorphism from $H^{-\frac{1}{2}}(\mathscr{C})$ onto $H^{\frac{1}{2}}(\mathscr{C})$, if $\operatorname{Cap}(\mathscr{C}) \neq 1$ (see [37, Theorem 8.16]). Under this condition, one can identify via this isomorphism any density $\hat{q} \in \widehat{H}(\mathscr{C})$ with the trace

$$
q:=\mathrm{S}_{\mathscr{C}} \hat{q} \in H(\mathscr{C}) .
$$

This identification will be systematically used throughout the paper, using the notation with (respectively without) a hat on the density like quantities (respectively on traces). This isomorphism turns out to be an isometry provided the spaces $\widehat{H}(\mathscr{C})$ and $H(\mathscr{C})$ are endowed with the following inner products:

Definition 2.4 For all $\hat{q}, \hat{p} \in \widehat{H}(\mathscr{C})$, we set:

$$
\langle\hat{q}, \hat{p}\rangle_{-\frac{1}{2}, \mathscr{C}}=\langle q, p\rangle_{\frac{1}{2}, \mathscr{C}}=\langle\hat{q}, p\rangle_{-\frac{1}{2}, \frac{1}{2}, \mathscr{C}} \cdot
$$

According to 2.3), the inner products introduced in Definition 2.4 are related to the Dirichlet energy of the single layer potential through the following identities:

$$
\|\hat{q}\|_{-\frac{1}{2}, \mathscr{C}}^{2}=\|q\|_{\frac{1}{2}, \mathscr{C}}^{2}=\int_{\mathbb{R}^{2}}\left|\nabla\left(\mathscr{S}_{\mathscr{C}} \hat{q}\right)\right|^{2} \mathrm{~d} x, \quad \forall \hat{q} \in \widehat{H}(\mathscr{C}) .
$$

We also need in the sequel the following orthogonal projections.

Definition 2.5 Let $\Pi_{\mathscr{C}}$ and $\widehat{\Pi}_{\mathscr{C}}$ denote respectively the orthogonal projections from $H^{\frac{1}{2}}(\mathscr{C})$ into $H(\mathscr{C})$ and from $H^{-\frac{1}{2}}(\mathscr{C})$ into $\widehat{H}(\mathscr{C})$.

In particular, we have the following unique decompositions:

$$
\begin{aligned}
\forall \hat{q} \in H^{-\frac{1}{2}}(\mathscr{C}): & \hat{q}=\langle\hat{q}, 1\rangle \hat{\mathrm{e}}_{\mathscr{C}}+\hat{q}_{0}, & \hat{q}_{0}:=\widehat{\Pi}_{\mathscr{C}} \hat{q} \in \widehat{H}(\mathscr{C}), \\
\forall q \in H^{\frac{1}{2}}(\mathscr{C}): & q=\left\langle\hat{\mathrm{e}}_{\mathscr{C}}, q\right\rangle 1+q_{0}, & q_{0}:=\Pi_{\mathscr{C}} q \in H(\mathscr{C}) .
\end{aligned}
$$

Definition 2.6 We denote by $\operatorname{Tr}_{\mathscr{C}}$ the classical trace operator (valued into $H^{\frac{1}{2}}(\mathscr{C})$ ), and by $\operatorname{Tr}_{\mathscr{C}}^{0}$ when it is left-composed with the orthogonal projection onto $H(\mathscr{C}): \operatorname{Tr}_{\mathscr{C}}^{0}:=\Pi_{\mathscr{C}} \operatorname{Tr}_{\mathscr{C}}$. 
Let us conclude this preliminary section by a useful characterization of the chosen norm on $H(\mathscr{C})$. Classically, we define the quotient weighted Sobolev space:

$$
W_{0}^{1}\left(\mathbb{R}^{2}\right)=\left\{u \in \mathcal{D}^{\prime}\left(\mathbb{R}^{2}\right): \rho u \in L^{2}\left(\mathbb{R}^{2}\right), \nabla u \in\left(L^{2}\left(\mathbb{R}^{2}\right)\right)^{2}\right\} / \mathbb{R},
$$

where the weight is given by

$$
\rho(x):=\left(\sqrt{1+|x|^{2}} \log \left(2+|x|^{2}\right)\right)^{-1}, \quad x \in \mathbb{R}^{2},
$$

and where the quotient means that functions of $W_{0}^{1}\left(\mathbb{R}^{2}\right)$ are defined up to an additive constant. This space is a Hilbert space once equipped with the inner product:

$$
\langle u, v\rangle_{W_{0}^{1}\left(\mathbb{R}^{2}\right)}:=\int_{\mathbb{R}^{2}} \nabla u \cdot \nabla v \mathrm{~d} x .
$$

In particular, according to 2.3$), \mathscr{S}_{\mathscr{C}} \hat{q} \in W_{0}^{1}\left(\mathbb{R}^{2}\right)$ if and only if $q \in H(\mathscr{C})$, and moreover

$$
\|\hat{q}\|_{-\frac{1}{2}, \mathscr{C}}=\|q\|_{\frac{1}{2}, \mathscr{C}}=\left\|\mathscr{S}_{\mathscr{C}} \hat{q}\right\|_{W_{0}^{1}\left(\mathbb{R}^{2}\right)}, \quad \forall q \in H(\mathscr{C}) .
$$

Lemma 2.7 For every $q \in H(\mathscr{C})$, we have

$$
\|q\|_{\frac{1}{2}, \mathscr{C}}=\inf \left\{\|u\|_{W_{0}^{1}\left(\mathbb{R}^{2}\right)}: u \in W_{0}^{1}\left(\mathbb{R}^{2}\right) \text { and } \operatorname{Tr}_{\mathscr{C}}^{0} u=q\right\} .
$$

The infimum is a minimum which is uniquely achieved by $u=\mathscr{S}_{\mathscr{C}} \hat{q}$.

Proof : Given $q \in H(\mathscr{C})$, let us consider the orthogonal decomposition:

$$
W_{0}^{1}\left(\mathbb{R}^{2}\right)=\left\langle\mathscr{S}_{\mathscr{C}} \hat{q}\right\rangle \oplus\left\langle\mathscr{S}_{\mathscr{C}} \hat{q}\right\rangle^{\perp} .
$$

Let $u \in W_{0}^{1}\left(\mathbb{R}^{2}\right)$ be such that $\operatorname{Tr}_{\mathscr{C}}^{0} u=q$. Writing $u$ in the form $u=\lambda \mathscr{S}_{\mathscr{C}} \hat{q}+v$ with $\lambda \in \mathbb{R}$ and $v \in\left\langle\mathscr{S}_{\mathscr{C}} \hat{q}\right\rangle^{\perp}$, and taking the projected trace on $\mathscr{C}$ we get:

$$
q=\lambda q+\operatorname{Tr}_{\mathscr{C}}^{0} v \quad \text { in } H(\mathscr{C})
$$

Forming now the duality product with $\hat{q}$ and taking into account that: $\left\langle\hat{q}, \operatorname{Tr}_{\mathscr{C}}^{0} v\right\rangle_{-\frac{1}{2}, \frac{1}{2}, \mathscr{C}}=\left\langle\mathscr{S}_{\mathscr{C}} \hat{q}, v\right\rangle_{W_{0}^{1}\left(\mathbb{R}^{2}\right)}=$ 0 , we deduce that $\lambda=1$. Since we have now

$$
\|u\|_{W_{0}^{1}\left(\mathbb{R}^{2}\right)}^{2}=\|q\|_{\frac{1}{2}, \mathscr{C}}^{2}+\|v\|_{W_{0}^{1}\left(\mathbb{R}^{2}\right)}^{2}
$$

with $v$ such that $\operatorname{Tr}_{\mathscr{C}}^{0} v=0$, the conclusion follows. 


\subsection{Boundary interaction and single layer potential}

In this section, we are interested in quantifying the Dirichlet energy variation between $\mathscr{S}_{\Gamma} \hat{q}$ and $\mathscr{S}_{\gamma} \hat{p}$ where $p=\operatorname{Tr}_{\gamma}^{0} \mathscr{S}_{\Gamma} \hat{q}$ (i.e. $p$ is the trace of the single layer potential $\mathscr{S}_{\Gamma} \hat{q}$ on $\gamma$ ).

Definition 2.8 We define the boundary interaction operators $\mathrm{K}_{\Gamma}^{\gamma}$ and $\mathrm{K}_{\gamma}^{\Gamma}$ between $\Gamma$ and $\gamma$ by:

$$
\mathrm{K}_{\Gamma}^{\gamma}: q \in H(\Gamma) \longmapsto \operatorname{Tr}_{\gamma}^{0}\left(\mathscr{S}_{\Gamma} \hat{q}\right) \in H(\gamma), \quad \mathrm{K}_{\gamma}^{\Gamma}: p \in H(\gamma) \longmapsto \operatorname{Tr}_{\Gamma}^{0}\left(\mathscr{S}_{\gamma} \hat{p}\right) \in H(\Gamma),
$$

where $\operatorname{Tr}_{\gamma}^{0}$ and $\operatorname{Tr}_{\Gamma}^{0}$ are given in Definition 2.6.

The next result shows that $\operatorname{Tr}_{\Gamma}^{0}$ can be replaced by $\operatorname{Tr}_{\Gamma}$ in the definition of $\mathrm{K}_{\gamma}^{\Gamma}$ :

Lemma 2.9 If $p \in H(\gamma)$, then $q:=\operatorname{Tr}_{\Gamma}\left(\mathscr{S}_{\gamma} \hat{p}\right)$ belongs to $H(\Gamma)$.

Proof : Let $p$ and $q$ be given as in the statement of the lemma and let us define the function $w:=$ $\left(w_{i}, w_{e}\right)$ in $H_{\ell o c}^{1}\left(\mathbb{R}^{2}\right)$ by setting: $w_{i}=\mathscr{S}_{\Gamma} \hat{q}$ in $\Omega$ and $w_{e}=\mathscr{S}_{\gamma} \hat{p}$ in $\mathbb{R}^{2} \backslash \bar{\Omega}$. According to (2.3), the function $w$ has finite Dirichlet energy since $p \in H(\gamma)$. Thanks to $(2.1)$, we see that $w_{e}(x)=O\left(|x|^{-1}\right)$ at infinity, and this allows us to obtain the following classical integral representation formula for every $x \in \mathbb{R}^{2} \backslash \bar{\Omega}$ (see for instance [40, p. 182] or [17, Lemma 3.5])

$$
\begin{aligned}
w_{e}(x) & =\left\langle\partial_{n} G(x-\cdot), w_{e}\right\rangle_{\frac{1}{2}, \Gamma}-\left\langle\partial_{n} w_{e}, G(x-\cdot)\right\rangle_{-\frac{1}{2}, \frac{1}{2}, \Gamma} \\
0 & =\left\langle\partial_{n} G(x-\cdot), w_{i}\right\rangle_{-\frac{1}{2}, \frac{1}{2}, \Gamma}-\left\langle\partial_{n} w_{i}, G(x-\cdot)\right\rangle_{-\frac{1}{2}, \frac{1}{2}, \Gamma}
\end{aligned}
$$

Since $\operatorname{Tr}_{\Gamma} w_{i}=\operatorname{Tr} r_{\Gamma} w_{e}=q$, we get by subtracting these identities that:

$$
w_{e}(x)=\langle\hat{r}, G(x-\cdot)\rangle_{-\frac{1}{2}, \frac{1}{2}, \Gamma}=\mathscr{S}_{\Gamma} \hat{r}(x), \quad x \in \mathbb{R}^{2} \backslash \bar{\Omega},
$$

where the density $\hat{r}:=\partial_{n} w_{i}-\partial_{n} w_{e}$ belongs to $\widehat{H}(\Gamma)$ since, as already mentioned, $w$ has finite Dirichlet energy. Taking the trace on $\Gamma$, we deduce from the above relation that $q=r \in H(\Gamma)$ and the proof is complete.

Proposition 2.10 The operators $\mathrm{K}_{\Gamma}^{\gamma}$ and $\mathrm{K}_{\gamma}^{\Gamma}$ are compact, one-to-one and dense-range operators. Moreover, for every functions $q \in H(\Gamma)$ and $p \in H(\gamma)$, we have:

$$
\left\langle\mathrm{K}_{\Gamma}^{\gamma} q, p\right\rangle_{\frac{1}{2}, \gamma}=\left\langle q, \mathrm{~K}_{\gamma}^{\Gamma} p\right\rangle_{\frac{1}{2}, \Gamma} \cdot
$$

Proof: The compactness follows from the regularity of the single layer potential away from the boundary, combined to [41, Proposition 13.5.8].

Addressing the symmetry property, consider $q \in L^{\infty}(\Gamma) \cap H(\Gamma)$ and $p \in L^{\infty}(\gamma) \cap H(\gamma)$. We can write that:

$$
\left\langle\mathrm{K}_{\Gamma}^{\gamma} q, p\right\rangle_{\frac{1}{2}, \gamma}=\int_{\gamma} \int_{\Gamma} G(x-y) \hat{q}(y) \hat{p}(x) \mathrm{d} \sigma_{y} \mathrm{~d} \sigma_{x}=\int_{\Gamma} \int_{\gamma} G(x-y) \hat{q}(x) \hat{p}(y) \mathrm{d} \sigma_{y} \mathrm{~d} \sigma_{x}=\left\langle\mathrm{K}_{\gamma}^{\Gamma} p, q\right\rangle_{\frac{1}{2}, \Gamma},
$$

and the conclusion follows by density. 
Assume now that $q \in H(\Gamma)$ is such that $\mathrm{K}_{\Gamma}^{\gamma} q=0$. By the unique continuation property for harmonic functions, it means that $\mathscr{S}_{\Gamma} \hat{q}$ is constant in $\Omega$ and hence that $q=0$. Since

$$
\overline{\operatorname{Ran} \mathrm{K}_{\gamma}^{\Gamma}}=\operatorname{Ker} \mathrm{K}_{\Gamma}^{\gamma},
$$

we get the density result and the proof is completed.

Proposition 2.11 The norms of the operators $\mathrm{K}_{\Gamma}^{\gamma}$ and $\mathrm{K}_{\gamma}^{\Gamma}$ are strictly less that 1.

Proof : According to Lemma 2.7 we have, for every $q \in H(\Gamma)$ :

$$
\left\|\mathrm{K}_{\Gamma}^{\gamma} q\right\|_{\frac{1}{2}, \gamma}=\inf \left\{\|u\|_{W_{0}^{1}\left(\mathbb{R}^{2}\right)}: u \in W_{0}^{1}\left(\mathbb{R}^{2}\right) \text { and } \operatorname{Tr}_{\gamma}^{0} u=\operatorname{Tr}_{\gamma}^{0}\left(\mathscr{S}_{\Gamma} \hat{q}\right)\right\} .
$$

We deduce that $\left\|\mathrm{K}_{\Gamma}^{\gamma} q\right\|_{\frac{1}{2}, \gamma} \leqslant\left\|\mathscr{S}_{\Gamma} \hat{q}\right\|_{W_{0}^{1}\left(\mathbb{R}^{2}\right)}=\|q\|_{\frac{1}{2}, \Gamma}$ and the norm of $\mathrm{K}_{\Gamma}^{\gamma}$ is no greater than 1 .

The operator $\mathrm{K}_{\Gamma}^{\gamma}$ being compact, its norm is achieved by some $q_{\Gamma} \in H(\Gamma)$. If $\left\|\mathrm{K}_{\Gamma}^{\gamma} q_{\Gamma}\right\|_{\frac{1}{2}, \gamma}=\left\|q_{\Gamma}\right\|_{\frac{1}{2}, \Gamma}$, we would have, according to Lemma 2.7 .

$$
\mathscr{S}_{\gamma} \hat{q}_{\gamma}=\mathscr{S}_{\Gamma} \hat{q}_{\Gamma}, \quad \text { in } \mathbb{R}^{2},
$$

where $q_{\gamma}:=\mathrm{K}_{\Gamma}^{\gamma} q_{\Gamma}$. This identity implies that $\hat{q}_{\Gamma}=\left[\partial_{n}\left(\mathscr{S}_{\gamma} \hat{q}_{\gamma}\right)\right]_{\Gamma}=0$, yielding the expected contradiction.

\section{$2.3 \quad$ Integral formulation and well-posedness}

Let us go back to Problem (1.2). Without loss of generality, let us assume from now on that the diameter of $\Omega$ is less than 1 (otherwise, it suffices to rescale the problem), which implies in particular that $\operatorname{Cap}(\Gamma)<1$ and $\operatorname{Cap}(\gamma)<1$ (see [40, p. 143] and references therein).

Proposition 2.12 For every $f \in H(\Gamma)$, denote by $\left(u^{f}, c^{f}\right) \in H^{1}(\Omega \backslash \bar{\omega}) \times \mathbb{R}$ the unique solution of System (1.2). The function $u^{f}$ can be represented as a superposition of single layer potentials as follows:

$$
u^{f}=\mathscr{S}_{\gamma} \hat{p}+\mathscr{S}_{\Gamma} \hat{q},
$$

where $\hat{p} \in \widehat{H}(\gamma)$ and $\hat{q} \in H^{-\frac{1}{2}}(\Gamma)$ solve the following system of coupled integral equations on the boundaries $\gamma$ and $\Gamma$ :

$$
\begin{array}{ll}
p+\operatorname{Tr}_{\gamma}\left(\mathscr{S}_{\Gamma} \hat{q}\right)=c^{f} & \text { on } \gamma \\
\operatorname{Tr}_{\Gamma}\left(\mathscr{S}_{\gamma} \hat{p}\right)+q=f & \text { on } \Gamma .
\end{array}
$$

Proof : It is a consequence of [37, Theorem 8.16] that the unique solution to System (1.2) can be written as a superposition of two single layer potentials respectively supported on $\Gamma$ and $\gamma$ and respectively associated with the densities $(\hat{p}, \hat{q}) \in H^{-\frac{1}{2}}(\gamma) \times H^{-\frac{1}{2}}(\Gamma)$, as in 2.4. It only remains to verify that $p$ is in fact in $\widehat{H}(\gamma)$, i.e. that $\langle\hat{p}, 1\rangle_{-\frac{1}{2}, \frac{1}{2}, \gamma}=0$. This is a straightforward consequence of (1.2d) and the jump relation for the normal derivative of the single layer potential. 


\section{The reconstruction formula}

Going back to the DtN operator $\Lambda_{\gamma}$ of problem 1.2 , and due to $1.2 \mathrm{~d}$, we have by Green's formula

$$
\left\langle\partial_{n} u^{f}, 1\right\rangle_{-\frac{1}{2}, \frac{1}{2}, \Gamma}=-\left\langle\partial_{n} u^{f}, 1\right\rangle_{-\frac{1}{2}, \frac{1}{2}, \gamma}=0,
$$

which shows that $\Lambda_{\gamma}$ is valued in $\widehat{H}(\Gamma)$. Considering data $f \in H(\Gamma)$, we can thus define the DtN operator $\Lambda_{\gamma}$ as follows:

$$
\Lambda_{\gamma}: f \in H(\Gamma) \longmapsto \partial_{n} u^{f} \in \widehat{H}(\Gamma) .
$$

In the case where $\omega=\varnothing$, we will denote respectively by $u_{0}^{f}$ and $\Lambda_{0}$ the solution $u^{f}$ and the $\operatorname{DtN} \Lambda_{\gamma}$. Note that we have in particular

$$
u_{0}^{f}=\mathscr{S}_{\Gamma} \hat{f} .
$$

\subsection{Factorization of the DtN map}

Theorem 3.1 The two following bounded linear operators in $H(\Gamma)$ :

$$
\mathrm{R}:=\mathrm{S}_{\Gamma}\left(\Lambda_{\gamma}-\Lambda_{0}\right) \quad \text { and } \quad \mathrm{K}:=\mathrm{K}_{\gamma}^{\Gamma} \mathrm{K}_{\Gamma}^{\gamma},
$$

satisfy the following equivalent identities:

$$
\mathrm{R}=(\mathrm{Id}-\mathrm{K})^{-1} \mathrm{~K}, \quad \mathrm{~K}=(\mathrm{Id}+\mathrm{R})^{-1} \mathrm{R} .
$$

Proof : Given $f$ in $H(\Gamma)$, let $(\hat{p}, \hat{q}) \in \widehat{H}(\gamma) \times H^{-\frac{1}{2}}(\Gamma)$ be the solution of System (2.5). According to Lemma 2.9. $\operatorname{Tr}_{\Gamma}\left(\mathscr{S}_{\gamma} \hat{p}\right) \in H(\Gamma)$ and hence $\operatorname{Tr}_{\Gamma}\left(\mathscr{S}_{\gamma} \hat{p}\right)=\mathrm{K}_{\gamma}^{\Gamma} p$. Since $f \in H(\Gamma)$, we deduce from $\left.2.5 \mathrm{~b}\right)$ that $q=f-\mathrm{K}_{\gamma}^{\Gamma} p \in H(\Gamma)$. Applying the projector $\Pi_{\gamma}$ to 2.5a), we obtain the following system:

$$
\begin{array}{ll}
p+\mathrm{K}_{\Gamma}^{\gamma} q=0 & \text { on } \gamma \\
\mathrm{K}_{\gamma}^{\Gamma} p+q=f & \text { on } \Gamma .
\end{array}
$$

Eliminating $p$, it follows that $(\mathrm{Id}-\mathrm{K}) q=f$ and hence $(\mathrm{Id}-\mathrm{K})(q-f)=\mathrm{K} f$. The operator $\mathrm{K}$ being a contraction (see Propositon 2.11), we end up with:

$$
q-f=(\mathrm{Id}-\mathrm{K})^{-1} \mathrm{~K} f
$$

On the other hand, we have

$$
\left(\Lambda_{\gamma}-\Lambda_{0}\right) f=\left.\partial_{n}\left(\mathscr{S}_{\Gamma} \hat{q}+\mathscr{S}_{\gamma} \hat{p}\right)\right|_{\Gamma}-\left.\partial_{n}\left(\mathscr{S}_{\Gamma} \hat{f}\right)\right|_{\Gamma}
$$

But outside $\Gamma$, the two single layer potentials $\mathscr{S}_{\Gamma} \hat{f}$ and $\mathscr{S}_{\Gamma} \hat{q}+\mathscr{S}_{\gamma} \hat{p}$ both solve the well-posed Dirichlet exterior boundary value problem:

$$
\begin{aligned}
-\Delta u & =0 & & \text { in } \mathbb{R}^{2} \backslash \bar{\Omega}, \\
u & =f & & \text { on } \Gamma, \\
u & =O\left(|x|^{-1}\right) & & |x| \rightarrow+\infty .
\end{aligned}
$$


Hence $\left(\mathscr{S}_{\Gamma} \hat{q}+\mathscr{S}_{\gamma} \hat{p}\right)=\mathscr{S}_{\Gamma} \hat{f}$ in $\mathbb{R}^{2} \backslash \bar{\Omega}$, and in particular we can rewrite 3.5 as

$$
\left(\Lambda_{\gamma}-\Lambda_{0}\right) f=\left[\partial_{n}\left(\mathscr{S}_{\Gamma} \hat{q}+\mathscr{S}_{\gamma} \hat{p}\right)\right]_{\Gamma}-\left[\partial_{n}\left(\mathscr{S}_{\Gamma} \hat{f}\right)\right]_{\Gamma}=\hat{q}-\hat{f},
$$

where the last equality follows from the jump relation (2.2). Comparing this relation and (3.4), we obtain that $\mathrm{S}_{\Gamma}\left(\Lambda_{\gamma}-\Lambda_{0}\right) f=(\mathrm{Id}-\mathrm{K})^{-1} \mathrm{~K} f$, which is exactly the first relation in $(3.2)$. The second relation follows easily.

The first equation in (3.2) can be seen as a factorization of the (known) DtN operator $\Lambda_{\gamma}-\Lambda_{0}$ in terms of the (unknown) boundary interaction operator $\mathrm{K}_{\Gamma}^{\gamma}$ and $\mathrm{K}_{\gamma}^{\Gamma}$. Similarly, the second equation in (3.2) can be seen as a factorization of the boundary interaction operator $\mathrm{K}=\mathrm{K}_{\gamma}^{\Gamma} \mathrm{K}_{\Gamma}^{\gamma}$ in terms of the measurement operator $\mathrm{R}$ (which is entirely determined by the perturbed and unperturbed DtN maps and by the exterior boundary $\Gamma$ ). Using Proposition 2.10, it is worth reformulating this second equation in a variational form:

$$
\left\langle\mathrm{K}_{\Gamma}^{\gamma} f, \mathrm{~K}_{\Gamma}^{\gamma} g\right\rangle_{\frac{1}{2}, \gamma}=\left\langle(\mathrm{Id}+\mathrm{R})^{-1} \mathrm{R} f, g\right\rangle_{\frac{1}{2}, \Gamma}, \quad \forall f, g \in H(\Gamma) .
$$

This identity constitutes the first step towards the reconstruction of the unknown boundary $\gamma$. Indeed, the bilinear form $\left\langle\mathrm{K}_{\Gamma}^{\gamma} \cdot \mathrm{K}_{\Gamma}^{\gamma} \cdot\right\rangle_{\frac{1}{2}, \gamma}$ turns out to encode the geometry of the inclusion, as shown in the next section.

\subsection{Harmonic polynomials and GPST}

Throughout the paper, we identify $x=\left(x_{1}, x_{2}\right)$ in $\mathbb{R}^{2}$ with the complex number $z=x_{1}+i x_{2}$.

Definition 3.2 For every $m \geqslant 1$, we define the harmonic polynomials of degree $m$ :

$$
P_{1}^{m}(x)=\operatorname{Re}\left(z^{m}\right) \quad \text { and } \quad P_{2}^{m}(x)=\operatorname{Im}\left(z^{m}\right) .
$$

We define as well

$$
Q_{1, \Gamma}^{m}(x):=P_{\ell}^{m}(x)+c_{1, \Gamma}^{m} \quad \text { and } \quad Q_{2, \Gamma}^{m}(x):=P_{\ell}^{m}(x)+c_{2, \Gamma}^{m}
$$

where the constant $c_{\ell, \Gamma}^{m} \in \mathbb{R}, \ell=1,2$, are chosen such that the trace of $Q_{\ell, \Gamma}^{m}$ on $\Gamma$ belongs to $H(\Gamma)$.

Finally, we set

$$
Q_{\Gamma}^{m}:=Q_{1, \Gamma}^{m}+i Q_{2, \Gamma}^{m} .
$$

The crucial point about these polynomials $Q_{\ell, \Gamma}^{m}, \ell=1,2$, lies in the fact that since they are harmonic, we have

$$
\mathrm{K}_{\Gamma}^{\gamma}\left(\left.Q_{\ell, \Gamma}^{m}\right|_{\Gamma}\right)=\left.Q_{\ell, \gamma}^{m}\right|_{\gamma},
$$

and hence, using these harmonic polynomials $Q_{\ell, \Gamma}^{m}$ in formula (3.6) (and using for simplicity the same notation for the functions and their traces on the boundaries $\gamma$ and $\Gamma$ ), we obtain that for all $m, m^{\prime} \geqslant 1$ and all $\ell, \ell^{\prime}=1,2$ :

$$
\left\langle\mathrm{K}_{\Gamma}^{\gamma} Q_{\ell, \Gamma}^{m}, \mathrm{~K}_{\Gamma}^{\gamma} Q_{\ell^{\prime}, \Gamma}^{m^{\prime}}\right\rangle_{\frac{1}{2}, \Gamma}=\left\langle Q_{\ell, \gamma}^{m}, Q_{\ell^{\prime}, \gamma}^{m^{\prime}}\right\rangle_{\frac{1}{2}, \gamma}
$$

Remark 3.3 The quantities $\left\langle Q_{\ell, \gamma}^{m}, Q_{\ell^{\prime}, \gamma}^{m^{\prime}}\right\rangle_{\frac{1}{2}, \gamma}$ are strongly connected with the so-called Generalized Pólya-Szegö Tensors (GPST) appearing in the high-order asymptotic expansion of the DtN map for small inclusions (see for instance the recent papers by Ammari et al. [5, 4] and references therein). Our definition is somehow different from theirs, as they use real polynomials $x^{m}$, while we use harmonic polynomials. 


\subsection{From the GPST to the geometry of the cavity: an explicit inversion formula}

In this section, we are going to see that the quantities $\left\langle Q_{\ell, \gamma}^{m}, Q_{\ell^{\prime}, \gamma}^{m^{\prime}}\right\rangle_{\frac{1}{2}, \gamma}$ for $m, m^{\prime} \geqslant 1$ and $\ell, \ell^{\prime}=1,2$, which can be deduced from the measurements (see (3.6), contain all the necessary information to reconstruct the cavity. We can even say more: the geometric information of $\gamma$ is actually redundant in the GPST. As we shall see, the knowledge of the quantities $\left\langle Q_{\gamma}^{m}, Q_{\gamma}^{1}\right\rangle_{\frac{1}{2}, \gamma}$ and $\left\langle Q_{\gamma}^{m}, \overline{Q_{\gamma}^{1}}\right\rangle_{\frac{1}{2}, \gamma}$ suffices to reconstruct the cavity. More precisely, assume that the geometry of $\gamma$ is described through the conformal mapping

$$
\phi: z \mapsto a_{1} z+a_{0}+\sum_{m \leqslant-1} a_{m} z^{m},
$$

that maps the exterior of the unit disk $D$ onto the exterior of $\omega$ (see the book of Pommerenke [38, p. 5] for the existence of such a mapping). In particular, $t \in]-\pi, \pi] \mapsto \phi\left(e^{i t}\right)$ provides a parameterization of $\gamma$. Notice that in this description, $\left|a_{1}\right|$ is the logarithmic capacity of $\gamma$ and can be chosen such that $a_{1}>0$. The coefficient $a_{0}$ is the conformal center of $\omega$. With these notation, we have the following result.

Theorem 3.4 Let $\left(Q_{\gamma}^{m}\right)_{m \geqslant 1}$ be the complex harmonic polynomials defined by (3.7)-(3.8). Define the two following sequences of complex numbers $(1 \leqslant m \leqslant+\infty)$ :

$$
\begin{aligned}
\mu_{m} & :=\frac{1}{2}\left\langle Q_{\gamma}^{m}, \overline{Q_{\gamma}^{1}}\right\rangle_{\frac{1}{2}, \gamma}=\frac{1}{2}\left\langle Q_{\Gamma}^{m},(\mathrm{Id}+\mathrm{R})^{-1} \mathrm{R} \overline{Q_{\Gamma}^{1}}\right\rangle_{\frac{1}{2}, \Gamma}, \\
\nu_{m} & :=\frac{1}{2}\left\langle Q_{\gamma}^{m}, Q_{\gamma}^{1}\right\rangle_{\frac{1}{2}, \gamma}=\frac{1}{2}\left\langle Q_{\Gamma}^{m},(\mathrm{Id}+\mathrm{R})^{-1} \mathrm{R} Q_{\Gamma}^{1}\right\rangle_{\frac{1}{2}, \Gamma},
\end{aligned}
$$

with $\mathrm{R}:=\mathrm{S}_{\Gamma}\left(\Lambda_{\gamma}-\Lambda_{0}\right)$. Then, $\mu_{1}>0$ and we have the explicit formulae:

$$
\begin{aligned}
a_{1} & =\left(\frac{\mu_{1}}{2 \pi}\right)^{\frac{1}{2}} a_{0}=\frac{\mu_{2}}{2 \mu_{1}} \\
a_{-m} & =\mu_{1}^{-\frac{m}{2}} \sum_{\alpha \in \mathrm{A}_{m}} C_{\alpha}\left(\frac{\mu_{2}}{\mu_{1}}\right)^{\alpha_{0}} \nu_{1}^{\alpha_{1}} \nu_{2}^{\alpha_{2}} \ldots \nu_{m}^{\alpha_{m}}, \quad m \geqslant 1,
\end{aligned}
$$

where

$$
\mathrm{A}_{m}:=\left\{\alpha \in \mathbb{N}^{m+1}: \alpha_{0}+2 \alpha_{1}+3 \alpha_{2}+\ldots+(m+1) \alpha_{m}=(m+1), \alpha_{0} \neq m+1\right\}
$$

and

$$
C_{\alpha}:=\frac{(-1)^{|\alpha|+1}}{2^{\alpha_{0}} m} \frac{(2 \pi)^{\frac{m}{2}-\left(\alpha_{1}+\cdots+\alpha_{m}\right)}}{1^{\alpha_{1}} 2^{\alpha_{2}} \ldots m^{\alpha_{m}}} .
$$

The rest of this section is devoted to the proof of this result.

To simplify the forthcoming computation, we complete the sequence of complex numbers $\left(a_{k}\right)_{k \leqslant 1}$ by setting $a_{k}=0$ for $k \geqslant 2$. We denote $a_{k}^{n}(n \in \mathbb{N}, k \in \mathbb{Z})$ the $k$ th coefficients of the Laurent's series of $\phi^{n}$ :

$$
a_{k}^{n}=\sum_{|\alpha|=k} a_{\alpha_{1}} a_{\alpha_{2}} \ldots a_{\alpha_{n}}
$$

where the sum ranges over all the multi-indices $\alpha=\left(\alpha_{1}, \ldots, \alpha_{n}\right) \in \mathbb{Z}^{n}$ whose length $|\alpha|=\alpha_{1}+\ldots+\alpha_{n}$ is equal to $k$. We also introduce the quantities:

$$
\mu^{m, m^{\prime}}:=\frac{1}{2}\left\langle Q_{\gamma}^{m}, \overline{Q_{\gamma}^{m^{\prime}}}\right\rangle_{\frac{1}{2}, \gamma}=\frac{1}{2}\left\langle Q_{\Gamma}^{m},(\mathrm{Id}+\mathrm{R})^{-1} \mathrm{R} \overline{Q_{\Gamma}^{m^{\prime}}}\right\rangle_{\frac{1}{2}, \Gamma}
$$




$$
\nu^{m, m^{\prime}}:=\frac{1}{2}\left\langle Q_{\gamma}^{m}, Q_{\gamma}^{m^{\prime}}\right\rangle_{\frac{1}{2}, \gamma}=\frac{1}{2}\left\langle Q_{\Gamma}^{m},(\mathrm{Id}+\mathrm{R})^{-1} \mathrm{R} Q_{\Gamma}^{m^{\prime}}\right\rangle_{\frac{1}{2}, \Gamma},
$$

so that

$$
\mu_{m}=\mu^{m, 1} \quad \text { and } \quad \nu_{m}=\nu^{m, 1} .
$$

Lemma 3.5 Denoting, for every $m \geqslant 1$ :

$$
\phi_{+}^{m}(z)=\sum_{k \geqslant 1} a_{k}^{m} z^{k} \quad \text { and } \quad \phi_{-}^{m}(z)=\sum_{k \leqslant-1} a_{k}^{m} z^{k}
$$

the following identities hold true:

$$
\mu^{m, m^{\prime}}=\int_{-\pi}^{\pi} \overline{e^{i t}\left(\phi_{+}^{m^{\prime}}\right)^{\prime}\left(e^{i t}\right)} \phi^{m}\left(e^{i t}\right) \mathrm{d} t \quad \text { and } \quad \nu^{m, m^{\prime}}=\int_{-\pi}^{\pi} e^{i t}\left(\phi_{+}^{m^{\prime}}\right)^{\prime}\left(e^{i t}\right) \phi^{m}\left(e^{i t}\right) \mathrm{d} t .
$$

Proof : Let $m, m^{\prime} \geqslant 1$ and $\ell=1,2$ be fixed. For the sake of simplicity, we drop in this proof the dependence with respect to $\gamma$ and we denote $Q_{\ell, \gamma}^{m}$ simply by $Q_{\ell}^{m}$ ). We aim to compute the quantity:

$$
\left\langle Q_{\ell}^{m}, Q_{\ell}^{m^{\prime}}\right\rangle_{\frac{1}{2}, \gamma}=\left\langle\widehat{Q}_{\ell}^{m}, Q_{\ell}^{m^{\prime}}\right\rangle_{-\frac{1}{2}, \frac{1}{2}, \gamma}
$$

To do so, we recall that from the jump relation (2.2), we have $\widehat{Q}_{\ell}^{m}=\left[\partial_{n} U_{\ell}^{m}\right]_{\gamma}$, where $U_{\ell}^{m}:=\mathscr{S}_{\gamma} \widehat{Q}_{\ell}^{m}$. Let us denote by $U_{e, \ell}^{m}$ and $U_{i, \ell}^{m}$ the restrictions of $U_{\ell}^{m}$ respectively to $\mathbb{R}^{2} \backslash \bar{\omega}$ and $\omega$.

We know that $U_{e, l}^{m}$ solves the following exterior Dirichlet boundary problem:

$$
\begin{aligned}
-\Delta U_{e, \ell}^{m} & =0 & & \text { in } \mathbb{R}^{2} \backslash \bar{\omega} \\
U_{e, \ell}^{m} & =Q_{\ell}^{m} & & \text { on } \gamma, \\
U_{e, \ell}^{m}(x) & =O\left(|x|^{-1}\right) & & \text { as }|x| \rightarrow+\infty .
\end{aligned}
$$

The functions $u_{e, \ell}^{m}:=U_{e, \ell}^{m}(\phi)$ are harmonic in $\mathbb{R}^{2} \backslash \bar{D}(D$ denotes the unit disk) and satisfy:

$$
u_{e, 1}^{m}(x)=\operatorname{Re}\left(\phi^{m}(z)\right)+c_{1}^{m} \quad \text { and } \quad u_{e, 2}^{m}(x)=\operatorname{Im}\left(\phi^{m}(z)\right)+c_{2}^{m} .
$$

We can easily compute the constants $c_{1}^{m}$ and $c_{2}^{m}$ by writing that

$$
c_{1}^{m}+i c_{2}^{m}=-\int_{\gamma}\left(P_{1}^{m}(x)+i P_{2}^{m}(x)\right) \hat{\mathrm{e}}_{\gamma}(x) \mathrm{d} \sigma_{x}=-\int_{-\pi}^{\pi} \phi^{m}\left(e^{i t}\right)\left|\phi^{\prime}\left(e^{i t}\right)\right| \hat{\mathrm{e}}_{\gamma}\left(e^{i t}\right) \mathrm{d} t .
$$

But we know (from direct computations or from [25, Theorem 17.3.3]) that $\hat{\mathrm{e}}_{\gamma}\left(e^{i t}\right)=1 /\left(2 \pi\left|\phi^{\prime}\left(e^{i t}\right)\right|\right)$. It follows that $c_{1}^{m}+i c_{2}^{m}=-a_{0}^{m}$ and we have, on the boundary of $D$ :

$$
u_{e, 1}^{m}(x)=\frac{1}{2}\left[\phi^{m}(z)+\overline{\phi^{m}(z)}\right]-\operatorname{Re}\left(a_{0}^{m}\right) \quad \text { and } \quad u_{e, 2}^{m}(x)=-\frac{i}{2}\left[\phi^{m}(z)-\overline{\phi^{m}(z)}\right]-\operatorname{Im}\left(a_{0}^{m}\right) .
$$

This can be rewritten, using the identity $\bar{z}=1 / z$ on $\partial D$, as:

$$
\begin{aligned}
& u_{e, 1}^{m}(x)=\frac{1}{2}\left[\overline{\phi_{+}^{m}}\left(z^{-1}\right)+\phi_{-}^{m}(z)+\overline{\overline{\phi_{+}^{m}}}\left(z^{-1}\right)+\phi_{-}^{m}(z)\right] \\
& u_{e, 2}^{m}(x)=-\frac{i}{2}\left[-\overline{\phi_{+}^{m}}\left(z^{-1}\right)+\phi_{-}^{m}(z)-\left(-\overline{\overline{\phi_{+}^{m}}\left(z^{-1}\right)+\phi_{-}^{m}(z)}\right)\right] \text {. }
\end{aligned}
$$


These expressions lead us to introduce the following functions:

$$
\begin{aligned}
& w_{1}^{m}(z)=\overline{\phi_{+}^{m}}\left(z^{-1}\right)+\phi_{-}^{m}(z)=\phi^{m}(z)-a_{0}^{m}+\lambda_{1}^{m}(z) \\
& w_{2}^{m}(z)=-\overline{\phi_{+}^{m}}\left(z^{-1}\right)+\phi_{-}^{m}(z)=\phi^{m}(z)-a_{0}^{m}+\lambda_{2}^{m}(z),
\end{aligned}
$$

where

$$
\lambda_{1}^{m}(z)=\overline{\phi_{+}^{m}}\left(z^{-1}\right)-\phi_{+}^{m}(z) \quad \text { and } \quad \lambda_{2}^{m}(z)=-\overline{\phi_{+}^{m}}\left(z^{-1}\right)-\phi_{+}^{m}(z) .
$$

The functions $w_{1}^{m}$ and $w_{2}^{m}$ are holomorphic in $\mathbb{C} \backslash \bar{D}$ and:

$$
u_{e, 1}^{m}=\operatorname{Re}\left(w_{1}^{m}\right) \quad \text { and } \quad u_{e, 2}^{m}=\operatorname{Im}\left(w_{2}^{m}\right) \quad \text { on } \partial D .
$$

For every $X=\left(X_{1}, X_{2}\right) \in \mathbb{R}^{2}$ identified with $Z=X_{1}+i X_{2} \in \mathbb{C}$, we have:

$$
\nabla U_{e, 1}^{m}(\phi(z)) \cdot X=\operatorname{Re}\left[\left(w_{1}^{m}\right)^{\prime}(z) Z / \phi^{\prime}(z)\right] \quad \text { and } \quad \nabla U_{e, 2}^{m}(\phi(z)) \cdot X=\operatorname{Im}\left[\left(w_{2}^{m}\right)^{\prime}(z) Z / \phi^{\prime}(z)\right] .
$$

On $\gamma$, the outer unit normal vector is parameterized by $t \in\left[-\pi, \pi\left[\longmapsto e^{i t} \phi^{\prime}\left(e^{i t}\right) /\left|\phi^{\prime}\left(e^{i t}\right)\right|\right.\right.$, and therefore, for every $m^{\prime} \geqslant 1$ :

$$
\int_{\gamma} \partial_{n} U_{e, 1}^{m}(x) Q_{1}^{m^{\prime}}(x) \mathrm{d} \sigma_{x}=\int_{-\pi}^{\pi} \operatorname{Re}\left[e^{i t}\left(w_{1}^{m}\right)^{\prime}\left(e^{i t}\right)\right] \operatorname{Re}\left[\phi^{m^{\prime}}\left(e^{i t}\right)\right] \mathrm{d} t .
$$

On the other hand, $U_{i, \ell}^{m}$ solves the following interior problem:

$$
\begin{aligned}
-\Delta U_{i, \ell}^{m} & =0 & & \text { in } \omega \\
U_{i, \ell}^{m} & =Q_{\ell}^{m} & & \text { on } \gamma,
\end{aligned}
$$

whose unique solution is merely $U_{i, \ell}^{m}=Q_{\ell}^{m}$, so that:

$$
\int_{\gamma} \partial_{n} U_{i, 1}^{m}(x) Q_{1}^{m^{\prime}}(x) \mathrm{d} \sigma_{x}=\int_{-\pi}^{\pi} \operatorname{Re}\left[e^{i t}\left(\phi^{m}\right)^{\prime}\left(e^{i t}\right)\right] \operatorname{Re}\left[\phi^{m^{\prime}}\left(e^{i t}\right)\right] \mathrm{d} t .
$$

Gathering now (3.16), 3.17) and taking into account the expressions (3.14), we infer that:

$$
\left\langle Q_{1}^{m}, Q_{1}^{m^{\prime}}\right\rangle_{\frac{1}{2}, \gamma}=\int_{\gamma}\left[\partial_{n} U_{1}^{m}(x)\right]_{\gamma} Q_{1}^{m^{\prime}}(x) \mathrm{d} \sigma_{x}=-\int_{-\pi}^{\pi} \operatorname{Re}\left[e^{i t}\left(\lambda_{1}^{m}\right)^{\prime}\left(e^{i t}\right)\right] \operatorname{Re}\left[\phi^{m^{\prime}}\left(e^{i t}\right)\right] \mathrm{d} t .
$$

Notice now that, on $\partial D$, we have:

$$
\operatorname{Re}\left[e^{i t}\left(\lambda_{1}^{m}\right)^{\prime}\left(e^{i t}\right)\right]=-\left[\overline{e^{i t}\left(\phi_{+}^{m}\right)^{\prime}\left(e^{i t}\right)}+e^{i t}\left(\phi_{+}^{m}\right)^{\prime}\left(e^{i t}\right)\right]=-2 \operatorname{Re}\left[e^{i t}\left(\phi_{+}^{m}\right)^{\prime}\left(e^{i t}\right)\right],
$$

and therefore, 3.18 can be rewritten as:

$$
\left\langle Q_{1}^{m}, Q_{1}^{m^{\prime}}\right\rangle_{\frac{1}{2}, \gamma}=2 \int_{-\pi}^{\pi} \operatorname{Re}\left[e^{i t}\left(\phi_{+}^{m}\right)^{\prime}\left(e^{i t}\right)\right] \operatorname{Re}\left[\phi^{m^{\prime}}\left(e^{i t}\right)\right] \mathrm{d} t .
$$

Using similar arguments, lengthy but straightforward computations lead to:

$$
\begin{aligned}
\left\langle Q_{1}^{m}, Q_{2}^{m^{\prime}}\right\rangle_{\frac{1}{2}, \gamma} & =2 \int_{-\pi}^{\pi} \operatorname{Re}\left[e^{i t}\left(\phi_{+}^{m}\right)^{\prime}\left(e^{i t}\right)\right] \operatorname{Im}\left[\phi^{m^{\prime}}\left(e^{i t}\right)\right] \mathrm{d} t \\
& =2 \int_{-\pi}^{\pi} \operatorname{Im}\left[e^{i t}\left(\phi_{+}^{m}\right)^{\prime}\left(e^{i t}\right)\right] \operatorname{Re}\left[\phi^{m^{\prime}}\left(e^{i t}\right)\right] \mathrm{d} t
\end{aligned}
$$


and

$$
\left\langle Q_{2}^{m}, Q_{2}^{m^{\prime}}\right\rangle_{\frac{1}{2}, \gamma}=2 \int_{-\pi}^{\pi} \operatorname{Im}\left[e^{i t}\left(\phi_{+}^{m}\right)^{\prime}\left(e^{i t}\right)\right] \operatorname{Im}\left[\phi^{m^{\prime}}\left(e^{i t}\right)\right] \mathrm{d} t
$$

Formulae 3.11 follow.

Lemma 3.6 The following relations hold true:

$$
a_{1}=\sqrt{\frac{\mu_{1}}{2 \pi}} \quad \text { and } \quad a_{0}=\frac{\mu_{2}}{2 \mu_{1}} .
$$

Proof : For every $z \in \mathbb{C} \backslash \bar{D}$ (recall that $D$ denotes the unit disk), we have $\phi_{+}^{1}(z)=\phi_{+}(z)=a_{1} z$ and $\phi_{+}^{2}(z)=\left(a_{1}\right)^{2} z^{2}+2 a_{1} a_{0} z$, and hence

$$
z \phi_{+}^{\prime}(z)=a_{1} z \quad \text { and } \quad z\left(\phi_{+}^{2}\right)^{\prime}(z)=2\left(a_{1}\right)^{2} z^{2}+2 a_{1} a_{0} .
$$

Applying formulae (3.11), we obtain:

$$
\mu_{1}=2 \pi\left(a_{1}\right)^{2} \quad \text { and } \quad \mu_{2}=4 \pi\left(a_{1}\right)^{2} a_{0} .
$$

The conclusion of the lemma follows.

The conformal mapping $\phi^{-1}$ can be expanded as a Laurent's series having the form:

$$
\phi^{-1}(z)=b_{1} z+b_{0}+\sum_{k \leqslant-1} b_{k} z^{k}
$$

outside a disk $D^{\prime}$ centered at the origin and containing $\omega$. The complex coefficients $b_{k}(k \leqslant 1)$ can be deduced on the one hand from the coefficients $a_{k}$ of $\phi$, and on the other hand from the values of $\nu_{m}$, $(m \geqslant 1)$, as claimed in the following lemma:

Lemma 3.7 The following relations hold true:

$$
b_{1}=1 / a_{1} \quad \text { and } \quad b_{0}=-a_{0} / a_{1} .
$$

For every $m \geqslant 1$, we have:

$$
b_{-m}=-\frac{\nu_{m}}{2 \pi a_{1} m}=-\frac{1}{m} \sum_{|\beta|=-1} a_{\beta_{1}} \ldots a_{\beta_{m}}, \quad m \geqslant 1 .
$$

Proof : Identities (3.19) follow straightforwardly because $\phi$ and $\phi^{-1}$ are inverse mappings. Integrating by part the expression of $\nu_{m}=\nu^{m, 1}$ obtained from (3.11), we get:

$$
\nu_{m}=a_{1} \int_{-\pi}^{\pi} e^{i t} \phi^{m}\left(e^{i t}\right) \mathrm{d} t=-a_{1} m \int_{-\pi}^{\pi} e^{i t} \phi^{\prime}\left(e^{i t}\right) e^{i t} \phi^{m-1}\left(e^{i t}\right) \mathrm{d} t .
$$


Since $t \mapsto e^{i t}$ is a parameterization of $\partial D$, applying Cauchy's integral formula we get on the one hand

$$
\int_{-\pi}^{\pi} e^{i t} \phi^{m}\left(e^{i t}\right) \mathrm{d} t=-i \int_{\partial D} \phi^{m}(\xi) \mathrm{d} \xi=2 \pi a_{-1}^{m} .
$$

On the other hand, since $t \mapsto \phi\left(e^{i t}\right)$ is a parameterization of $\gamma$ and the function $\phi^{-1}$ being holomorphic in $\mathbb{C} \backslash \bar{\omega}$, we have:

$$
\int_{-\pi}^{\pi} e^{i t} \phi^{\prime}\left(e^{i t}\right) e^{i t} \phi^{m-1}\left(e^{i t}\right) \mathrm{d} t=-i \int_{\gamma} \phi^{-1}(\xi) \xi^{m-1} \mathrm{~d} \xi=-i \int_{\partial D^{\prime}} \phi^{-1}(\xi) \xi^{m-1} \mathrm{~d} \xi=2 \pi b_{-m} .
$$

Identity $(3.21)$ can thus be rewritten as:

$$
\nu_{m}=2 \pi a_{1} a_{-1}^{m}=-2 \pi a_{1} m b_{-m},
$$

and identity 3.20 follows according to 3.10 .

Using the above lemmas, we are in position to prove the main result of this section, namely Theorem 3.4

Proof of Theorem 3.4: Since $\phi$ and $\phi^{-1}$ play symmetric roles, we can exchange $a_{m}$ and $b_{m}$ in Formula 3.20 to obtain:

$$
a_{-m}=-\frac{1}{m} \sum_{|\beta|=-1} b_{\beta_{1}} \ldots b_{\beta_{m}}, \quad m \geqslant 1 .
$$

Reordering the terms of the above sum, we get that

$$
a_{-m}=-\frac{1}{m} \sum_{(\theta, \alpha) \in \mathrm{B}_{m}} b_{1}^{\theta} b_{0}^{\alpha_{0}} b_{-1}^{\alpha_{1}} \ldots b_{-m}^{\alpha_{m}}
$$

where $\mathbf{B}_{m}$ is the set of $(\theta, \alpha) \in \mathbb{N} \times \mathbb{N}^{m+1}$ such that

$$
\begin{aligned}
\theta+\alpha_{0}+\alpha_{1}+\cdots+\alpha_{m} & =m \\
\theta-\left(\alpha_{1}+2 \alpha_{2}+\cdots+m \alpha_{m}\right) & =-1 .
\end{aligned}
$$

Now, one can easily check that $(\theta, \alpha) \in \mathrm{B}_{m}$ if and only if $\alpha$ belongs to the set $\mathrm{A}_{m}$ defined by (3.9e) and $\theta=m-\left(\alpha_{0}+\alpha_{1}+\cdots+\alpha_{m}\right)$. Therefore, 3.22 also reads

$$
a_{-m}=-\frac{1}{m} \sum_{\alpha \in \mathrm{A}_{m}} b_{1}^{m-\left(\alpha_{0}+\alpha_{1}+\cdots+\alpha_{m}\right)} b_{0}^{\alpha_{0}} b_{-1}^{\alpha_{1}} \ldots b_{-m}^{\alpha_{m}}
$$

Using (3.19) and the first equality of (3.20) in the above relation, we obtain that

$$
a_{-m}=-\frac{1}{m} a_{1}^{-m} \sum_{\alpha \in \mathrm{A}_{m}}\left(-a_{0}\right)^{\alpha_{0}}\left(-\frac{\nu_{1}}{2 \pi}\right)^{\alpha_{1}} \ldots\left(-\frac{\nu_{m}}{2 \pi m}\right)^{\alpha_{m}}
$$

and the conclusion follows immediately. 


\subsection{About stability}

It is well-known that logarithmic stability is best possible for Calderón's inverse problem. In the particular case of cavities, this result is proved in [2, Theorem 4.1] where the error on the geometry (measured using the Hausdorff distance) is estimated in terms of the error of the DtN (measured in operator norm). However, as suggested by Alessandrini and Vessella [3], one can try to construct stable functionals, namely Lipschitz-continuous functions of the data carrying relevant information on the geometry of the obstacle. According to formula (3.9), each coefficient $a_{k}, k \leqslant 1$, yields an example of such functional. Actually, we can prove that each coefficient is not only a Lipschitz-continuous function of the data, but is analytic. Let us define the following open subspace of $\mathcal{L}(H(\Gamma))$ :

$$
\mathcal{U}_{\Gamma}=\left\{\mathrm{R} \in \mathcal{L}(H(\Gamma)): \mathrm{Id}+\mathrm{R} \text { invertible and } \mu_{1}(\mathrm{R})>0\right\},
$$

where $\mu_{1}(\mathrm{R}):=\left\langle Q_{\Gamma}^{1},(\mathrm{Id}+\mathrm{R})^{-1} \mathrm{R} \overline{Q_{\Gamma}^{1}}\right\rangle_{\frac{1}{2}, \Gamma}$.

Notice in particular that, for every Lipschitz Jordan curve $\gamma$, the continuous linear mapping $\mathrm{R}=$ $\mathrm{S}_{\Gamma}\left(\Lambda_{\gamma}-\Lambda_{0}\right)$ belongs to $\mathcal{U}_{\Gamma}$. We deduce straightforwardly the following analyticity result:

Theorem 3.8 On the open subset $\mathcal{U}_{\Gamma}$ of $\mathcal{L}(H(\Gamma))$ define the sequence of analytic functions $a_{k}: \mathcal{U}_{\Gamma} \rightarrow$ $\mathbb{C}(k \leqslant 1)$ as given by the formulae (3.9). If $\mathrm{R}=\mathrm{S}_{\Gamma}\left(\Lambda_{\gamma}-\Lambda_{0}\right)$ for some Lipschitz Jordan curve $\gamma$, a parameterization of $\gamma$ is given by:

$$
t \in]-\pi, \pi] \mapsto \sum_{k \leqslant 1} a_{k}(\mathrm{R}) e^{i k t} \in \mathbb{C} .
$$

\section{Numerical results}

We present in this section some numerical experiments meant to illustrate the feasibility of the proposed reconstruction method. For the sake of clarity, let us first sum up the different steps of the simple reconstruction algorithm:

1. Compute a numerical approximation of the operator $\mathrm{R}=\mathrm{S}_{\Gamma}\left(\Lambda_{\gamma}-\Lambda_{0}\right)$.

2. Fix an integer $M$ and compute for $1 \leqslant m \leqslant M$

$$
\begin{aligned}
& \mu_{m}:=\frac{1}{2}\left\langle Q_{\gamma}^{m}, \overline{Q_{\gamma}^{1}}\right\rangle_{\frac{1}{2}, \gamma}=\frac{1}{2}\left\langle Q_{\Gamma}^{m},(\mathrm{Id}+\mathrm{R})^{-1} \mathrm{R} \overline{Q_{\Gamma}^{1}}\right\rangle_{\frac{1}{2}, \Gamma} \\
& \nu_{m}:=\frac{1}{2}\left\langle Q_{\gamma}^{m}, Q_{\gamma}^{1}\right\rangle_{\frac{1}{2}, \gamma}=\frac{1}{2}\left\langle Q_{\Gamma}^{m},(\mathrm{Id}+\mathrm{R})^{-1} \mathrm{R} Q_{\Gamma}^{1}\right\rangle_{\frac{1}{2}, \Gamma} .
\end{aligned}
$$

3. Compute $\left(a_{-m}\right)_{-1 \leqslant m \leqslant M}$ via formulae $(3.9)$.

4. Plot the image of the unit circle by

$$
\phi_{M}(z)=a_{1} z+a_{0}+\sum_{1 \leqslant m \leqslant M} a_{-m} z^{-m}
$$

Let us give some details about the implementation. We use the finite dimensional approximation space spanned by the family $\mathcal{Q}_{\Gamma}^{M}:=\left\{Q_{\Gamma}^{m}, \overline{Q_{\Gamma}^{m}}, 1 \leqslant m \leqslant M\right\}$. We denote by $\mathbf{Q}_{\Gamma}$ the $2 M \times 2 M$ complex matrix whose entries are the $\langle f, g\rangle_{\frac{1}{2}, \Gamma}$, where $(f, g) \in \mathcal{Q}_{\Gamma}^{M} \times \mathcal{Q}_{\Gamma}^{M}$. Note that $\mathbf{Q}_{\Gamma}$ is nothing but the Generalized 
Polya-Szegö Tensor (GPST) associated to $\Gamma$. Obviously, a similar matrix $\mathbf{Q}_{\gamma}$ can be defined for the boundary $\gamma$. We denote by $\mathbf{R}$ the matrix whose entries are $\langle f, \operatorname{Rg}\rangle_{\frac{1}{2}, \Gamma}=\left\langle\left(\Lambda_{\gamma}-\Lambda_{0}\right) g, f\right\rangle_{-\frac{1}{2}, \frac{1}{2}, \Gamma}$, for $(f, g) \in \mathcal{Q}_{\Gamma}^{M} \times \mathcal{Q}_{\Gamma}^{M}$. With this notation, the reader can easily check that formula (3.6) admits the following discrete version:

$$
\mathbf{Q}_{\gamma} \simeq \widetilde{\mathbf{Q}}_{\gamma}:=\mathbf{Q}_{\Gamma}\left(\mathbf{Q}_{\Gamma}+\mathbf{R}\right)^{-1} \mathbf{R}
$$

This formula relates in a very simple way, through the measurement operator $\mathbf{R}$, the GPST of $\gamma$ to the GPST of $\Gamma$. In particular, the coefficients $\mu_{m}$ and $\nu_{m}$ are particular entries of $\frac{1}{2} \mathbf{Q}_{\gamma}$.

We consider now a test configuration in which $\Gamma$ is an ellipse centered at the origin and of major axis $[-1.9,1.9]$ and minor axis $[-1.1,1.1]$. The boundary $\gamma$ of the obstacle is parameterized by:

$$
t \in]-\pi, \pi] \mapsto \sum_{k=-7}^{1} a_{k} e^{i k t}
$$

where the complex coefficients $a_{k}$ are given in the following table:

\begin{tabular}{|c|c|c|c|c|c|c|c|c|}
\hline$a_{1}$ & $a_{0}$ & $a_{-1}$ & $a_{-2}$ & $a_{-3}$ & $a_{-4}$ & $a_{-5}$ & $a_{-6}$ & $a_{-7}$ \\
\hline 0.5 & -1 & 0.085 & $-0.06 i$ & -0.035 & $0.06 i$ & 0 & $-0.01 i$ & -0.005 \\
\hline
\end{tabular}

The data are generated using the Matlab Laplace boundary integral equation solver (for more information, see this link: IES). Taking $M=12$, we first show on Figure 2 the reconstructed cavity for exact data and using the eight coefficients $a_{1}, \ldots, a_{-6}$.

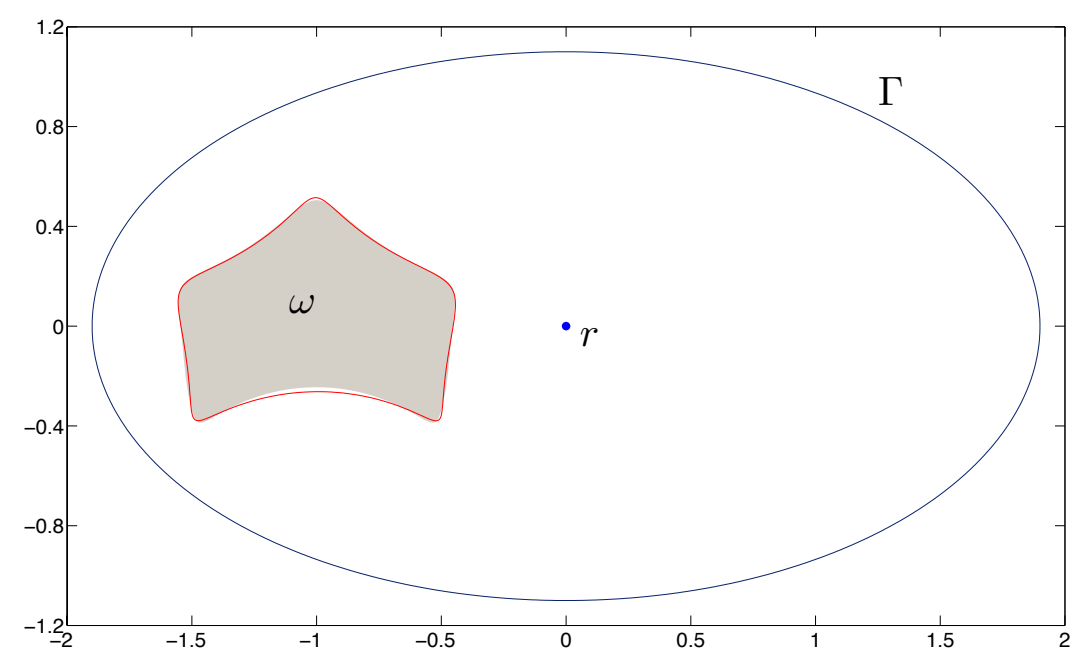

Figure 2: Typical configuration: reconstruction with $a_{1}, \ldots, a_{-6}$ (in red) and actual inclusion (in gray). The blue point stands for the position of the origin $r$.

Instead of using the harmonic polynomials $z^{n}$ in Definition 3.2, one can use the shifted harmonic polynomials $(z-r)^{n}$, for some given $r \in \mathbb{C}$. This additional parameter turns out to have some influence on the quality of the reconstructed cavities, as shown in Figure 3 . For instance, choosing $r$ in the neighborhood of -0.5 , one can recover the six coefficients $a_{1}, \ldots, a_{-4}$ with a relative error less than $2 \%$, while for $r=0$ this accuracy is achieved only for the coefficients $a_{1}, \ldots, a_{-2}$. 


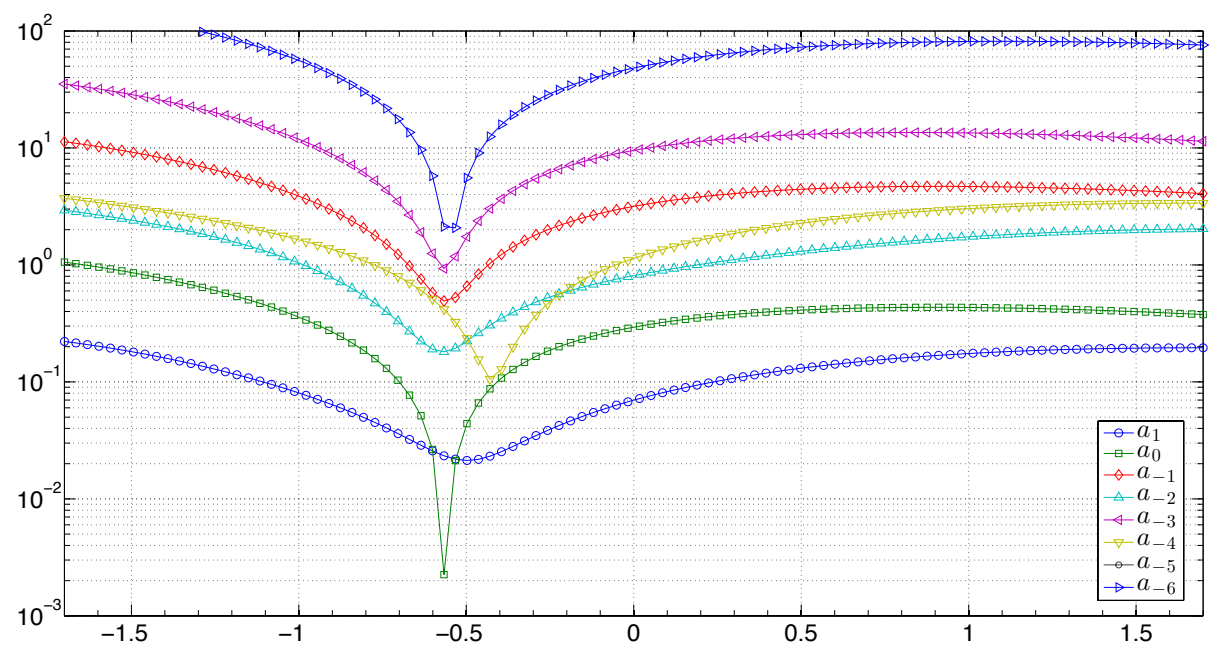

Figure 3: Relative error of the retrieved coefficients (in \%) with respect to the abscissa of $r$ (there is no relative error for the coefficient $a_{-5}$ because it is null).

Let us take now $r=-0.5$ and consider a more realistic configuration of noisy data. We generate a random matrix $\mathbf{N}$ having the same size as $\mathbf{R}$ and whose coefficients are uniformly distributed between -1 and 1. For $\delta=0.05,0.15,0.25,0.35$, we compute the matrix $\mathbf{R}^{N}$ whose coefficients are:

$$
R_{i j}^{N}=\left(1+\delta N_{i j}\right) R_{i j}, \quad 1 \leqslant i, j \leqslant 2 M,
$$

and we replace $\mathbf{R}$ by $\mathbf{R}^{N}$ in formula 4.1 .

We show on figures 47 examples of reconstructed cavities respectively with $5 \%, 15 \%, 25 \%$ and $35 \%$ of noise. The number of correctly recovered coefficients decreases with the level of noise and only those coefficients are used in the reconstruction. We plot on Figure 8 the dependence of the mean relative error with respect to the level of noise and we notice a good stability of the first three coefficients $a_{1}, a_{0}$ and $a_{-1}$.

Finally, we illustrate on Figure 9 the efficiency of the method for more complex geometries (non convex outer boundary $\Gamma$ and a non centered cavity). The choice of the parameter $r$ to obtain good reconstructions is not clear so far and this would need to be further investigated.

\section{A Appendix}

Consider problem (1.1) with a piecewise conductivity $\sigma(x)=1+(\alpha-1) 1_{\omega}(x)$ (where $1_{\omega}$ denotes the characteristic function of $\omega$ and $\alpha$ a positive constant).

Proposition A.1 For every $f \in H^{\frac{1}{2}}(\Gamma)$, System 1.2 admits a unique solution $\left(u^{f}, c^{f}\right) \in H^{1}(\Omega \backslash$ $\bar{\omega}) \times \mathbb{R}$. It is the unique pair that realizes:

$$
\min _{(u, c) \in H^{1}(\Omega \backslash \bar{\omega}) \times \mathbb{R}}\left\{\frac{1}{2} \int_{\Omega \backslash \bar{\omega}}|\nabla u|^{2} \mathrm{~d} x:\left.u\right|_{\Gamma}=f,\left.u\right|_{\gamma}=c\right\} .
$$

The function $u^{f}$ can be considered as a function of $H^{1}(\Omega)$ by setting $u^{f}=c^{f}$ in $\omega$. 


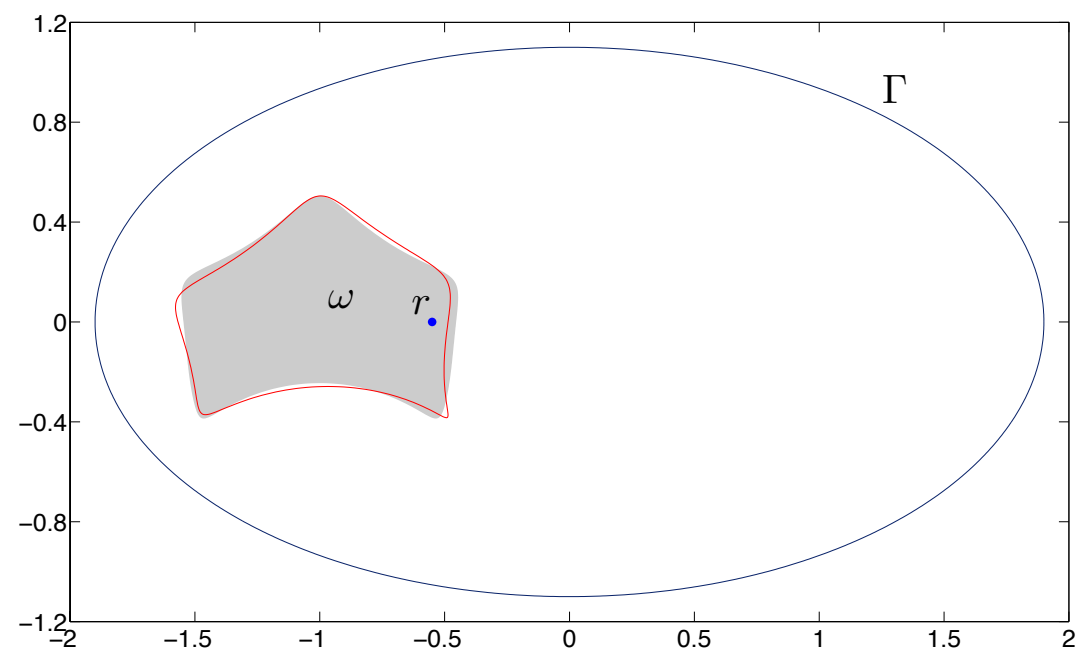

Figure 4: Reconstruction (in red) with $a_{1}, \ldots, a_{-4}$ and actual inclusion (in gray) with $5 \%$ noise.

For every $\alpha>0$, System (1.1) admits a unique solution $u_{\alpha}^{f} \in H^{1}(\Omega)$. This function achieves:

$$
\min _{u \in H^{1}(\Omega)}\left\{\frac{1}{2} \int_{\Omega \backslash \bar{\omega}}|\nabla u|^{2} \mathrm{~d} x+\frac{\alpha}{2} \int_{\omega}|\nabla u|^{2} \mathrm{~d} x:\left.u\right|_{\Gamma}=f\right\} .
$$

The following convergence result holds true for every $f \in H^{\frac{1}{2}}(\Gamma)$ :

$$
u_{\alpha}^{f} \rightarrow u^{f} \quad \text { in } H^{1}(\Omega) \text { as } \alpha \rightarrow+\infty .
$$

Proof : The minimization problem A.1 can be reformulated as:

$$
\min _{(w, c) \in H_{0}^{1}(\Omega \backslash \bar{\omega}) \times \mathbb{R}} \int_{\Omega \backslash \bar{\omega}}\left|\nabla\left(w+e^{f}+c v\right)\right|^{2} \mathrm{~d} x,
$$

where $e^{f}$ and $v$ are both harmonic in $\Omega \backslash \bar{\omega}$ with Dirichlet data $\left.e^{f}\right|_{\Gamma}=f,\left.e^{f}\right|_{\gamma}=0$ and $\left.v\right|_{\Gamma}=0,\left.v\right|_{\gamma}=1$. For every $w \in H_{0}^{1}(\Omega \backslash \bar{\omega})$, we get:

$$
\int_{\Omega \backslash \bar{\omega}}\left|\nabla\left(w+e^{f}+c v\right)\right|^{2} \mathrm{~d} x=\int_{\Omega \backslash \bar{\omega}}|\nabla w|^{2} \mathrm{~d} x+\int_{\Omega \backslash \bar{\omega}}\left|\nabla e^{f}\right|^{2} \mathrm{~d} x+c^{2} \int_{\Omega \backslash \bar{\omega}}|\nabla v|^{2} \mathrm{~d} x+2 c \int_{\gamma} \partial_{n} e^{f} \mathrm{~d} \sigma
$$

and therefore the minimum in $\mathrm{A} .3$ is unique and achieved for $w=0$ and

$$
c^{f}=-\int_{\gamma} \partial_{n} e^{f} \mathrm{~d} \sigma\left(\int_{\Omega \backslash \bar{\omega}}|\nabla v|^{2} \mathrm{~d} x\right)^{-1} .
$$

The corresponding fonction $u^{f}:=e^{f}+c^{f} v$ is the unique minimizer of problem A.1 , and can easily be shown to solve System (1.2). It is classical to verify that, reciprocally, every solution of System (1.2) provides a solution to the minimization problem A.1. 


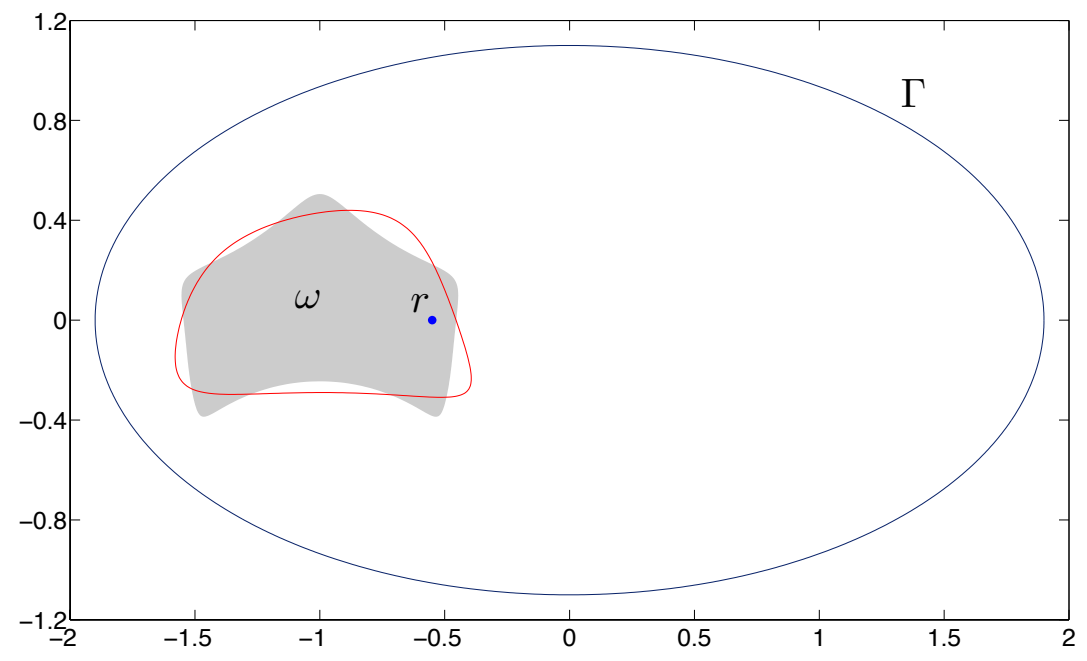

Figure 5: Reconstruction (in red) with $a_{1}, \ldots, a_{-4}$ and actual inclusion (in gray) with $15 \%$ noise.

Seeking the minimum of problem A.2 in the form $u=u^{f}+w$ with $w \in H_{0}^{1}(\Omega)$, we are led to consider the new, equivalent, minimization problem:

$$
\min _{w \in H_{0}^{1}(\Omega)} \int_{\Omega \backslash \bar{\omega}}|\nabla w|^{2} \mathrm{~d} x+\frac{\alpha}{2} \int_{\omega}|\nabla w|^{2} \mathrm{~d} x+\int_{\gamma} \partial_{n} u^{f} w \mathrm{~d} \sigma
$$

where we have used the fact that $u^{f}=c^{f}$ in $\omega$. The existence and uniqueness for such a problem is straightforward and we denote by $w_{\alpha}^{f}$ the minimizer. Introducing

$$
\bar{w}_{\alpha}^{f}=\frac{1}{\operatorname{mes}(\omega)} \int_{\omega} w_{\alpha}^{f} \mathrm{~d} x,
$$

and taking into account the condition $(1.2 \mathrm{~d})$, the last term in the right hand side can be rewritten as:

$$
\int_{\gamma} \partial_{n} u^{f} w_{\alpha}^{f} \mathrm{~d} \sigma=\int_{\gamma} \partial_{n} u^{f}\left(w_{\alpha}^{f}-\bar{w}_{\alpha}^{f}\right) \mathrm{d} \sigma .
$$

Invoking the Poincaré-Wirtinger inequality in $\omega$, we get the estimate:

$$
\left|\int_{\gamma} \partial_{n} u^{f} w_{\alpha}^{f} \mathrm{~d} \sigma\right| \leqslant C\left\|\nabla w_{\alpha}^{f}\right\|_{L^{2}(\omega)}
$$

where the constant $C>0$ depends only upon $\omega$. The minimum A.4 is negative $(w=0$ is an admissible function), whence we deduce that:

$$
\frac{\alpha}{2}\left\|\nabla w_{\alpha}^{f}\right\|_{L^{2}(\omega)} \leqslant C
$$

and therefore

$$
\left\|\nabla w_{\alpha}^{f}\right\|_{L^{2}(\omega)} \rightarrow 0 \quad \text { as } \quad \alpha \rightarrow+\infty .
$$

Remarking again that the minimum A.4 is negative and using the estimate (A.5) together with the convergence result (A.6), we deduce that:

$$
\left\|\nabla w_{\alpha}^{f}\right\|_{L^{2}(\Omega \backslash \bar{\omega})} \rightarrow 0 \quad \text { as } \quad \alpha \rightarrow+\infty
$$




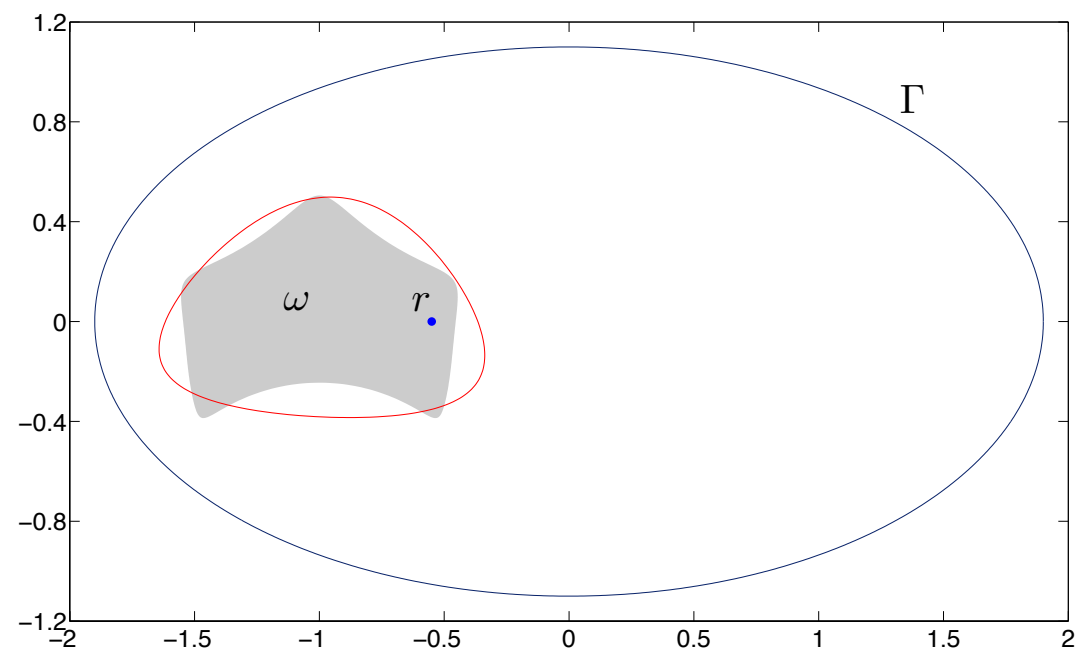

Figure 6: Reconstruction (in red) with $a_{1}, \ldots, a_{-2}$ and actual inclusion (in gray) with $25 \%$ noise.

and the proof is completed.

\section{References}

[1] I. Akduman And R. KRess, Electrostatic imaging via conformal mapping, Inverse Problems, 18 (2002), pp. 1659-1672.

[2] G. Alessandrini and L. Rondi, Optimal stability for the inverse problemof multiple cavities, Journal of Differential Equations, 176 (2001), pp. 356 - 386.

[3] G. Alessandrini and S. Vessella, Lipschitz stability for the inverse conductivity problem, Adv. in Appl. Math., 35 (2005), pp. 207-241.

[4] H. Ammari, Y. Deng, H. Kang, And H. Lee, Reconstruction of inhomogeneous conductivities via the concept of generalized polarization tensors, Ann. Inst. H. Poincaré Anal. Non Linéaire, 31 (2014), pp. 877-897.

[5] H. Ammari, J. Garnier, H. Kang, M. Lim, and S. Yu, Generalized polarization tensors for shape description, Numer. Math., 126 (2014), pp. 199-224.

[6] H. Ammari And H. KAng, Reconstruction of small inhomogeneities from boundary measurements, vol. 1846 of Lecture Notes in Mathematics, Springer-Verlag, Berlin, 2004.

[7] _ Generalized polarization tensors, inverse conductivity problems, and dilute composite materials: a review, in Inverse problems, multi-scale analysis and effective medium theory, vol. 408 of Contemp. Math., Amer. Math. Soc., Providence, RI, 2006, pp. 1-67.

[8] — Polarization and moment tensors, vol. 162 of Applied Mathematical Sciences, Springer, New York, 2007. 


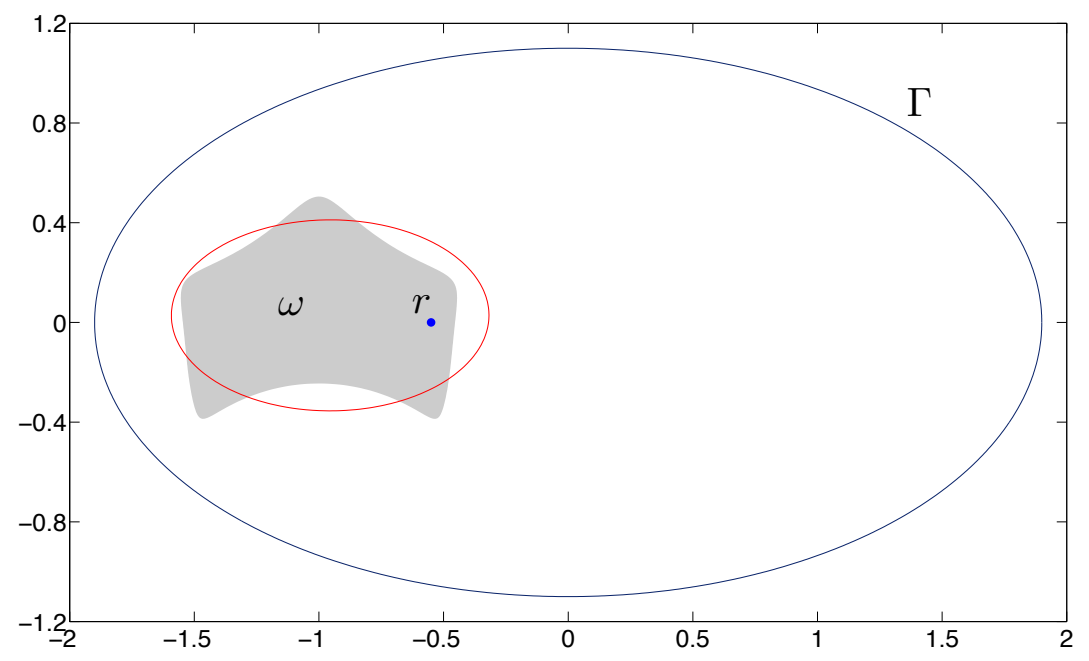

Figure 7: Reconstruction (in red) with $a_{1}, \ldots, a_{-1}$ and actual inclusion (in gray) with $35 \%$ noise.

[9] V. BACChELli, Uniqueness for the determination of unknown boundary and impedance with the homogeneous Robin condition, Inverse Problems, 25 (2009), pp. 015004, 4.

[10] L. Borcea, Electrical impedance tomography, Inverse Problems, 18 (2002), pp. R99-R136.

[11] L. Bourgeois And J. DARdÉ, A quasi-reversibility approach to solve the inverse obstacle problem, Inverse Probl. Imaging, 4 (2010), pp. 351-377.

[12] L. Bourgeois And J. DARdÉ, The "exterior approach" to solve the inverse obstacle problem for the Stokes system, Inverse Probl. Imaging, 8 (2014), pp. 23-51.

[13] M. Brühl And M. HANke, Numerical implementation of two noniterative methods for locating inclusions by impedance tomography, Inverse Problems, 16 (2000), pp. 1029-1042.

[14] F. CAKOnI AND R. KRess, Integral equation methods for the inverse obstacle problem with generalized impedance boundary condition, Inverse Problems, 29 (2013), pp. 015005, 19.

[15] A.-P. Calderón, On an inverse boundary value problem, in Seminar on Numerical Analysis and its Applications to Continuum Physics (Rio de Janeiro, 1980), Soc. Brasil. Mat., Rio de Janeiro, 1980, pp. 65-73.

[16] Y. Capdeboscq, J. Fehrenbach, F. De Gournay, and O. Kavian, Imaging by modification: numerical reconstruction of local conductivities from corresponding power density measurements, SIAM J. Imaging Sci., 2 (2009), pp. 1003-1030.

[17] M. Costabel and E. Stephan, A direct boundary integral equation method for transmission problems, J. Math. Anal. Appl., 106 (1985), pp. 367-413.

[18] K. Erhard and R. Potthast, A numerical study of the probe method, SIAM J. Sci. Comput., 28 (2006), pp. 1597-1612 (electronic). 


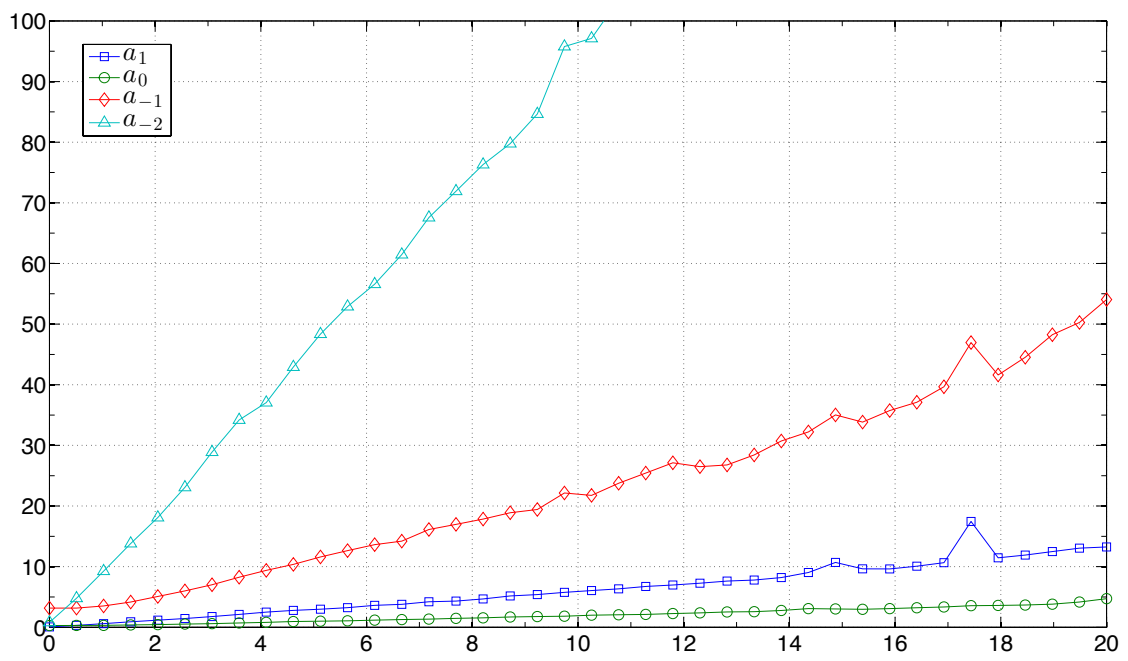

Figure 8: Relative error of the retrieved coefficients (in \%) with respect to the level of noise (in \%) of the data. The point $r$ is the center of the ellipse. Notice the stability of the conformal center $a_{0}$ and the logarithmic capacity $a_{1}$.

[19] A. Friedman AND M. Vogelius, Identification of small inhomogeneities of extreme conductivity by boundary measurements: a theorem on continuous dependence, Arch. Rational Mech. Anal., 105 (1989), pp. 299-326.

[20] H. Haddar and R. KRess, Conformal mappings and inverse boundary value problems, Inverse Problems, 21 (2005), pp. 935-953.

[21] H. Haddar AND R. KRess, Conformal mapping and an inverse impedance boundary value problem, J. Inverse Ill-Posed Probl., 14 (2006), pp. 785-804.

[22] H. Haddar and R. Kress, Conformal mapping and impedance tomography, Inverse Problems, 26 (2010), pp. 074002, 18.

[23] — A conformal mapping method in inverse obstacle scattering, Complex Var. Elliptic Equ., 59 (2014), pp. 863-882.

[24] M. Hanke And M. BRÜHL, Recent progress in electrical impedance tomography, Inverse Problems, 19 (2003), pp. S65-S90.

[25] E. Hille, Analytic function theory. Vol. II, Introductions to Higher Mathematics, Ginn and Co., Boston, Mass.-New York-Toronto, Ont., 1962.

[26] G. C. Hsiao and W. L. Wendland, Boundary integral equations, vol. 164 of Applied Mathematical Sciences, Springer-Verlag, Berlin, 2008.

[27] M. Ikehata, Reconstruction of the shape of the inclusion by boundary measurements, Comm. Partial Differential Equations, 23 (1998), pp. 1459-1474.

[28] — On reconstruction in the inverse conductivity problem with one measurement, Inverse Problems, 16 (2000), pp. 785-793. 

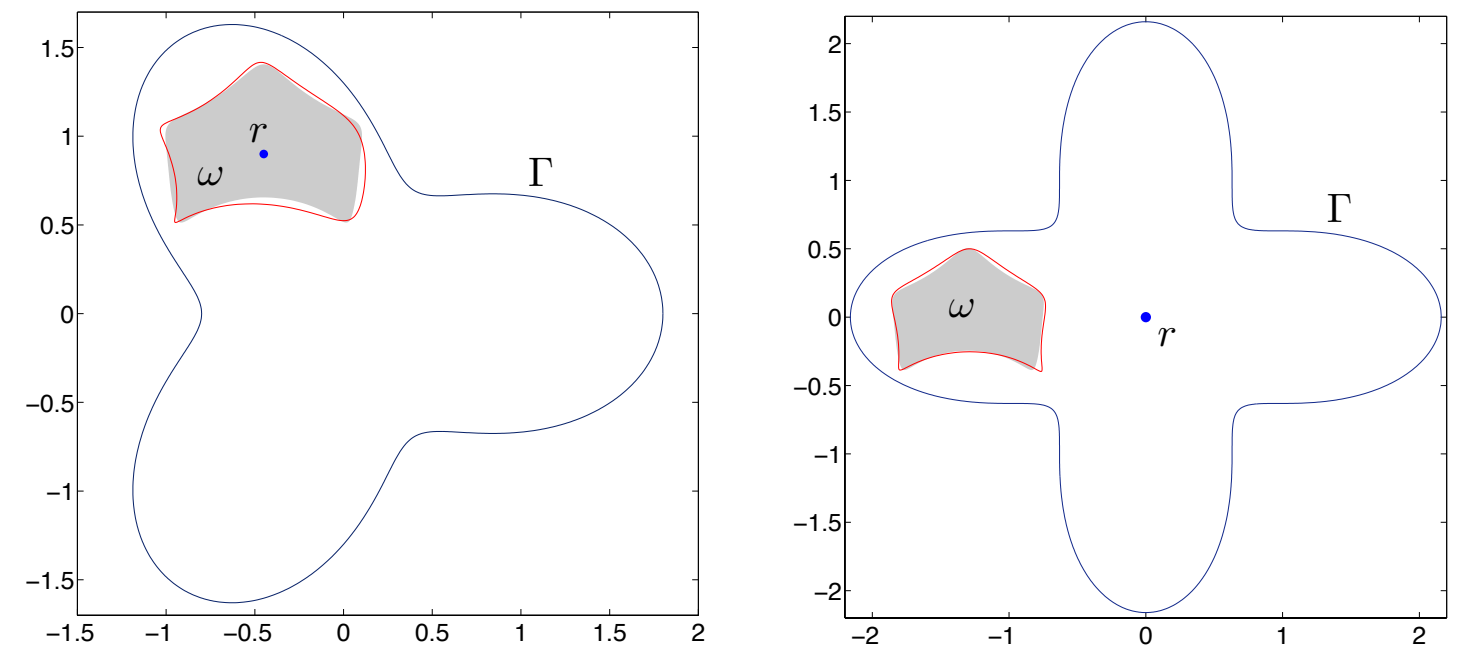

Figure 9: Other examples of reconstruction with more complex boundary $\Gamma$.

[29] M. IKEHATA, Reconstruction of the support function for inclusion from boundary measurements, J. Inverse Ill-Posed Probl., 8 (2000), pp. 367-378.

[30] M. Ikehata And S. Siltanen, Numerical method for finding the convex hull of an inclusion in conductivity from boundary measurements, Inverse Problems, 16 (2000), p. 1043.

[31] O. Ivanyshyn And R. KRess, Nonlinear integral equations for solving inverse boundary value problems for inclusions and cracks, J. Integral Equations Appl., 18 (2006), pp. 13-38.

[32] A. KIRsch, The factorization method for a class of inverse elliptic problems, Math. Nachr., 278 (2005), pp. 258-277.

[33] R. KRess, Inverse Dirichlet problem and conformal mapping, Math. Comput. Simulation, 66 (2004), pp. 255-265.

[34] R. KRess, Inverse problems and conformal mapping, Complex Var. Elliptic Equ., 57 (2012), pp. 301-316.

[35] R. KRess And W. Rundell, Nonlinear integral equations and the iterative solution for an inverse boundary value problem, Inverse Problems, 21 (2005), pp. 1207-1223.

[36] N. Mandache, Exponential instability in an inverse problem for the Schrödinger equation, Inverse Problems, 17 (2001), pp. 1435-1444.

[37] W. MCLean, Strongly elliptic systems and boundary integral equations, Cambridge University Press, Cambridge, 2000.

[38] C. Pommerenke, Boundary behaviour of conformal maps, vol. 299 of Grundlehren der Mathematischen Wissenschaften [Fundamental Principles of Mathematical Sciences], Springer-Verlag, Berlin, 1992.

[39] R. Potthast, A survey on sampling and probe methods for inverse problems, Inverse Problems, 22 (2006), pp. R1-R47. 
[40] O. Steinbach, Numerical approximation methods for elliptic boundary value problems, Springer, New York, 2008.

[41] M. TuCSNAK AND G. Weiss, Observation and control for operator semigroups, Birkhäuser Advanced Texts: Basler Lehrbücher. [Birkhäuser Advanced Texts: Basel Textbooks], Birkhäuser Verlag, Basel, 2009. 